\title{
COMPOSITION SERIES AND INTERTWINING OPERATORS FOR THE SPHERICAL PRINCIPAL SERIES. I
}

BY

\author{
KENNETH D. JOHNSON AND NOLAN R. WALLACH
}

\begin{abstract}
Let $G$ be a connected semisimple Lie group with finite center and let $K$ be a maximal compact subgroup. Let $\pi$ be a not necessarily unitary principal series representation of $G$ on the Hilbert space $H^{\pi}$. If $X^{\pi}$ denotes the space of $K$-finite vectors of $H^{\pi}, \pi$ induces a representation $\pi_{0}$ of $U(g)$, the enveloping algebra of the Lie algebra of $G$, on $X^{\pi}$.

In this paper, we determine when $\pi_{0}$ is irreducible, and if $\pi_{0}$ is not irreducible we determine the composition series of $X^{*}$ and the structure of the induced representations on the subquotients. Explicit computation of the intertwining operators for the different principal series representations are obtained and their relationship to polynomials defined by B. Kostant are obtained.
\end{abstract}

1. Introduction. Let $G$ be a connected semisimple Lie group with finite center. Let $G=K A N$ be an Iwasawa decomposition of $G$. That is, $K$ is a maximal compact subgroup of $G, A N$ is a solvable subgroup of $G$ with $A$ a vector group normalizing $N$, a simply connected nilpotent subgroup of $G$. Furthermore, the map of $K \times A \times N$ to $G$ given by $(k, a, n) \rightarrow k a n$ is an analytic diffeomorphism. Let $M$ be the centralizer of $A$ in $K$. Let $\mathfrak{g}, \mathrm{t}, \mathfrak{m}, \mathfrak{a}$, and $\mathfrak{n}$ denote the Lie algebras of $G, K, M, A$, and $N$ respectively. exp is a Lie isomorphism from a to $A$. We denote its inverse by $\log$. Finally, if $g \in G$,

$$
g=k(g) \exp H(g) n(g)
$$

where $k(g) \in K, H(g) \in a$ and $n(g) \in N$ and $k(g), H(g)$ and $n(g)$ are unique. We will use this notation throughout this paper.

If $\xi: M \rightarrow \mathcal{L}(H, H)$ is an irreducible unitary representation of $M$ and if $\nu \in \mathrm{a}_{\mathrm{C}}^{*}$ we define $H^{\xi, \nu}$ to be the space of all measurable functions $f: G \rightarrow H$ so that:

$$
f(\text { gman })=\xi(m)^{-1} e^{-v(\log a)} f(g) \text { for } g \text { in } G, m \text { in } M,
$$

$$
a \text { in } A, n \text { in } N \text {; }
$$

and

Received by the editors April 3, 1974 and, in revised form, July 16, 1975 and September 29, 1975.

AMS (MOS) subject classifications (1970). Primary 43A65, 22E43; Secondary 43A85, 33A75.

Key words and phrases. Principal series, intertwining operator, c-function, Euler operator, transvection, Poisson kernel, $K$-finite complementary series, spherical harmonics. 


$$
\int_{K}\|f(k)\|^{2} d k=\|f\|^{2}<\infty .
$$

Then $H^{\xi, \nu}$ is a Hilbert space relative to \|\| and left translation by elements of $G$ induces a continuous representation $\pi_{\xi, \nu}$ of $G$ on $H^{\xi, \nu}$. That is, if $f \in H^{\xi, \nu}$ and $g \in G,\left(\pi_{\xi, \nu}(g) f\right)(x)=f\left(g^{-1} x\right)$. The elements $\left(\pi_{\xi, \nu}, H^{\xi, \nu}\right)$ are called the principal series of $G$. If $X^{\xi, \nu}$ denotes the $K$-finite elements of $H^{\xi, \nu}$ then the elements of $X^{\xi, \nu}$ are in fact analytic functions on $G$. $\pi_{\xi, \nu}$ thus induces a representation of the universal enveloping algebra of $g$ on $X^{\xi, D}$. If $\xi=1$ is the trivial representation of $M$ (this gives the so-called spherical principal series of $G$ ) we shall denote $\pi_{1, v}, H^{1, \nu}$ and $X^{1, \nu}$ by $\pi_{\nu}, H^{\nu}$ and $X^{\nu}$ respectively. Of special interest to us will be the function $1, \in X^{\nu}$ which is 1 on $K$.

A natural question one may ask concerning these representations is what is the Jordan-Hölder series of $H^{\xi, \nu}$ under the action of $\pi_{\xi, \nu}$. A partial answer to this question was provided by Kostant [10] who determined which spherical principal series representations are irreducible.

This paper is the first of two papers dealing with the study of the composition series of the principal series of $G$. In this paper, we give complete descriptions of the composition series in the case that $G$ is a classical split rank 1 group and $\xi$ is the trivial representation of $M$. In the second paper [7], the problem is solved for the split rank 1 group of real type $F_{4}$. The two cases are separated since the classical groups may be dealt with in an almost uniform manner, whereas the study of $F_{4}$ involves an examination of the exceptional simple Jordan algebra and thus merits its own exposition.

In the course of our analysis, we give explicit formulas for the intertwining operators from $X^{\nu}$ to $X^{\nu^{\prime}}$ which we show for a general $G$ (not necessarily of split rank 1) may be expressed in terms of quotients of the " $P$ "--matrices defined by Kostant [10]. We also compute the "partial intertwining operators" from $X^{\nu}$ to $X^{\nu^{\prime}}$, and this enables us to determine which subquotients of the composition series of $X^{\nu}$ are unitarizable. In particular, we obtain Kostant's results [10] on the existence of a complementary series.

We also show how to use our results to compute Kostant's $P^{\gamma}$-matrices in the case when $G$ is of split rank 1 .

In addition to the results on the complementary series our results overlap with Kostant [10] on the irreducibility of the $H^{\nu}$ and with Knapp-Stein [9], Schiffmann [15] and Helgason [6] on intertwining operators. Our results on $S O(n, 1)$ can be found in Takahashi [17] and Vilenkin [18]. The results in the case $G=S U(2,1)$ have been announced by Stern [16]. In the case $G=$ $S L(2, \mathbf{R})(S U(1,1))$ our results can be found in Gelfand, Graev, Vilenkin [1] and Sally [14]. In the case $G=S L(2, \mathrm{C})(S O(3,1))$ the results can be found in Gelfand, et al. [1].

We now give a summary of the results in this paper. After a few brief remarks on the classical rank 1 groups we explicitly compute the $M$ fixed 
vectors of $X^{\nu}$ which we shall describe both as polynomials and as hypergeometric functions. We then explicitly compute the action of $\pi_{\nu}(H)$ (where $\mathbf{R} H=\mathfrak{a}$ ) on the $M$-fixed vectors. This allows us to compute the composition series and all the partial intertwining operators. We then show how to use our results to compute Kostant's $P^{\gamma}$-matrices in the case when $G$ is split rank 1 . Finally, we obtain some results on the non-square-integrability of most of the representations but not all which occur as subquotients.

2. The classical rank one symmetric spaces. Let $\mathbf{F}$ be one of the classical fields (that is $\mathbf{F}$ is either the reals, $\mathbf{R}$, the complexes, $\mathbf{C}$, or the quaternions, $\mathbf{H}$ ). Let $x \rightarrow \bar{x}$ be the standard involution on $\mathbf{F}$, and for $x$ in $\mathbf{F}$ set $|x|^{2}=x \bar{x}$.

Consider $F^{n+1}$ as a right vector space over $F$, and on $F^{n+1}$ define the quadratic form $Q\left(x_{1}, \ldots, x_{n+1}\right)=\left|x_{1}\right|^{2}+\cdots+\left|x_{n}\right|^{2}-\left|x_{n+1}\right|^{2}$. We define $G$ to be the group of all $\mathbf{F}$-linear transformations of $\mathbf{F}^{n+1}$ preserving $Q$ with the additional property that, if $\mathbf{F}=\mathbf{R}$ or $\mathbf{C}$ and $g \in G$, det $g=1$. Then if $\mathbf{F}=\mathbf{R}, \mathbf{C}$ or $\mathbf{H}$ respectively $G=S O(n, 1), S U(n, 1)$ or $S p(n, 1)$ respectively (these are the classical split rank 1 groups).

Let $e_{1}, \ldots, e_{n+1}$ be the standard basis of $\mathbf{F}^{n+1}$ and let $K$ be the group of all elements $k$ in $G$ so that $k\left(e_{n+1}\right) \in e_{n+1} \mathbf{F}$. Let $D^{n}$ be the unit ball in $F^{n}$, that is $D^{n}=\left\{\left.\left(x_{1}, \ldots, x_{n}\right)|| x_{1}\right|^{2}+\cdots+\left|x_{n}\right|^{2}<1\right\}$.

We now define an action of $G$ on $D^{n}$ as follows: If $z=z_{1} e_{1}+\cdots+z_{n} e_{n}$ and $g \in G$ then $g\left(z+e_{n+1}\right)=y_{1} e_{1}+\cdots+y_{n} e_{n}+y_{n+1} e_{n+1}$. Set

$$
g \cdot z=y_{1} y_{n+1}^{-1} e_{1}+\cdots+y_{n} y_{n+1}^{-1} e_{n}
$$

Since

$$
\left|y_{1}\right|^{2}+\cdots+\left|y_{n}\right|^{2}-\left|y_{n+1}\right|^{2}<0, \quad\left|z_{1}\right|^{2}+\cdots+\left|z_{n}\right|^{2}<1
$$

this implies that $g \cdot z$ is in $D^{n}$. Thus $g D^{n} \subset D^{n}$ and in fact the action of $g$ is actually $C^{\infty}$ in a neighborhood of $\overline{D^{n}}$.

We now show that $G$ acts transitively on $D^{n}$. If $u \in \mathrm{F}^{n}$ let $u^{*}(w)=\langle u, w\rangle$ $=\bar{u}_{1} w_{1}+\cdots+\bar{u}_{n} w_{n}$ for $w$ in $\mathbf{F}^{n}$. If $A$ is an $\mathbf{F}$-linear transformation of $\mathbf{F}^{n}$ and $u, v \in \mathbf{F}^{n}$ and $w \in \mathbf{F}$ define

$$
\left(g=\left[\begin{array}{cc}
A & u \\
v^{*} & w
\end{array}\right]\right)\left(\begin{array}{c}
z \\
z_{n+1}
\end{array}\right)=\left(\begin{array}{c}
A z+u z_{n+1} \\
v^{*}(z)+w z_{n+1}
\end{array}\right)
$$

for $z \in \mathbf{F}^{n}$ and $z_{n+1} \in \mathbf{F}$. Then $g$ defines an $\mathbf{F}$-linear transformation of $\mathbf{F}^{n+1}$.

If $z \in D^{n}$ set $\|z\|^{2}=\left|z_{1}\right|^{2}+\cdots+\left|z_{n}\right|^{2}$ and let $A[z]$ be the positive definite square root of $I+\left(z \otimes z^{*}\right) /\left(1-\|z\|^{2}\right)$ where $\left(z \otimes z^{*}\right)(w)=$ $z\left(z^{*}(w)\right) . A[z]$ is clearly well defined. Now set 


$$
T[z]=\left[\begin{array}{cc}
A[z] & \frac{z}{\left(1-\|z\|^{2}\right)^{1 / 2}} \\
\frac{z^{*}}{\left(1-\|z\|^{2}\right)^{1 / 2}} & \frac{1}{\left(1-\|z\|^{2}\right)^{1 / 2}}
\end{array}\right]
$$

Here we use the natural identification that if $u \in F^{n}$ so is $u^{*}$.

A simple check shows that $T[z]$ is in $G$ and that $T[z] \cdot 0=z$. Thus we see that $G$ acts transitively on $D^{n}$.

A simple examination of $G$ shows that if $g \in G, g$ is of the form $g=\left(\begin{array}{c}A \\ v^{*} \\ w\end{array}\right)$ where $A$ is an $n \times n$ matrix, $u, v \in \mathrm{F}^{n}$ and $w \in \mathrm{F}$, and $g^{-1}=\left(A_{-u^{*}}^{*} \overline{\bar{w}}^{-0}\right)$ where $A^{*}$ is the conjugate transpose of $A$. Thus, we see that $g$ consists of all matrices of the form

$$
X=\left(\begin{array}{cc}
A & u \\
u^{*} & w
\end{array}\right) \text { where }\left(\begin{array}{cc}
A & u \\
u^{*} & w
\end{array}\right)+\left(\begin{array}{cc}
A^{*} & -u \\
-u^{*} & \bar{w}
\end{array}\right)=0,
$$

and if $\mathbf{F}=\mathbf{C}$ we have the additional property that $\operatorname{tr} X=0$.

If we set $H=e_{1} \otimes e_{n+1}^{*}+e_{n+1} \otimes e_{1}^{*}$ we may then take $a=\mathbf{R} H$. With $a$ and hence $A$ determined, we now have that $M$ is the group of all matrices in $G$ of the form

$$
\left[\begin{array}{lll}
b & 0 & 0 \\
0 & B & 0 \\
0 & 0 & b
\end{array}\right]
$$

where $B$ is an $(n-1) \times(n-1)$ matrix and $|b|^{2}=1$. For $n$ we make the choice that $\mathfrak{n}$ is spanned by all matrices of the form

$$
\left[\begin{array}{ccc}
0 & z & 0 \\
-z^{*} & 0 & z^{*} \\
0 & z & 0
\end{array}\right]
$$

with $z \in F^{n-1}$ and of the form

$$
\left(\begin{array}{ccc}
w & 0 & -w \\
0 & 0 & 0 \\
w & 0 & -w
\end{array}\right)
$$

with $w \in \mathbf{F}$ and $w+\bar{w}=0$. Now this choice of $\mathrm{n}$ thus determines $N$.

Under the identification of $G / K$ with $D^{n}$ we see that $G / M A N=K / M$ may be identified with $S^{d n-1}$, the unit sphere in $F^{n}$ where $d=\operatorname{dim}_{R} \mathbf{F}$. Note that $M A N=\left\{g \in G \mid g \cdot e_{1}=e_{1}\right.$ where $G$ is considered as acting on $\left.\bar{D}^{n}\right\}$.

As $G$ acts on $K / M$ via left translation, $g \in G$ induces a linear map on the exterior forms of $K / M$ and we denote this map by $g^{*}$.

If $\omega$ is a $K$-invariant volume element on $K / M$, the Poisson kernel is the function $P: G / K \times K / M \rightarrow \mathrm{R}$ defined by 


$$
\left(\left(g^{-1}\right)^{*} \omega\right)_{b}=P(g K, b) \omega_{b} \text { for } g \in G \text { and } b \in K / M \text {. }
$$

From Harish-Chandra [21] we have that

$$
P(g K, k M)=\exp \left(-2 \rho H\left(g^{-1} k\right)\right)
$$

where for $H \in \mathfrak{a}, 2 \rho(H)=\operatorname{tr}$ ad $\left.H\right|_{\mathfrak{n}}$.

On $F^{n+1} \times F^{n+1}$ let $B$ be the $F$-valued bilinear form such that $B(x, y)=$ $\bar{x}_{1} y_{1}+\cdots+\bar{x}_{n} y_{n}-\bar{x}_{n+1} y_{n+1}$. Suppose that $g=k a_{t} n$ where $a_{t}=\exp t H$ and $H=e_{1} \otimes e_{n+1}^{*}+e_{n+1} \otimes e_{1}^{*}$. Then $2 \rho(H)=d(n+1)-2$ and $P(g K, 1 M)=e^{-(d(n+1)-1) t}$. A simple calculation shows that

$$
\left|B\left(g^{-1}\left(e_{n+1}\right), e_{1}+e_{n+1}\right)\right|^{2}=\left|B\left(a_{t}^{-1}\left(e_{n+1}\right), e_{1}+e_{n+1}\right)\right|^{2}=e^{2 t} \text {. }
$$

Thus we see that $P(g K, k M)=\left|B\left(g e_{n+1}, k\left(e_{1}+e_{n+1}\right)\right)\right|^{2-d(n+1)}$. Now using the fact that $g$ is of the form

$$
g=\left(\begin{array}{cc}
A & u \\
v^{*} & w
\end{array}\right) \text { with }|w|^{2}-\|u\|^{2}=1
$$

and that $g \cdot 0=u w^{-1}$ we obtain by a simple calculation that

$$
\left|B\left(g\left(e_{n+1}\right), k\left(e_{1}+e_{n+1}\right)\right)\right|^{2}=\frac{|1-\langle g \cdot 0, b\rangle|^{2}}{1-\|g \cdot 0\|^{2}}
$$

where $k M=b$ (that is, $k\left(e_{1}+e_{n+1}\right)=b^{\prime}+\alpha e_{n+1}$ and $\left.b=b^{\prime} \alpha^{-1}\right)$. Thus we obtain the following:

LEMMA 2.1.

$$
P(z, b)=\left(\frac{1-\|z\|^{2}}{|1-\langle z, b\rangle|^{2}}\right)^{d(n+1) / 2-1} .
$$

3. Spherical harmonics. Since our work in this paper will be infinitesimal we will actually be studying the representations $\left(\pi_{p}, X^{v}\right)$ of the enveloping algebra of $g$. We will therefore want an explicit description of the elements of $X^{\prime}$.

Since, as a $K$-representation, $X^{\nu}$ may be identified with the $K$-finite elements of $L^{2}(K / M)=L^{2}\left(S^{d n-1}\right)$, we see that determining the members of $X^{\nu}$ is the same as the determination of Peter-Weyl decomposition of $L^{2}\left(S^{d n-1}\right)$.

Let $\hat{K}$ denote the set of equivalence classes of irreducible finite dimensional representations of $K$, and if $\left(\pi_{\gamma}, V_{\gamma}\right) \in \gamma \in \hat{K}$ set $V_{\gamma}^{M}=\left\{v \in V_{\gamma} \mid \pi(m) v=v\right.$ for all $m$ in $M$ \}. Frobenius reciprocity combined with the Peter-Weyl theorem imply that the space of $K$-finite elements of $L^{2}\left(S^{d n-1}\right)$ decomposes into $\Sigma_{\gamma \in \hat{K}_{\gamma} V_{\gamma}}$ where $n_{\gamma}=\operatorname{dim} V_{\gamma}^{M}$. In the future we shall express this as $L^{2}\left(S^{d n-1}\right) \sim \Sigma_{\gamma \in \hat{K}^{n}} V_{\gamma}$. 
Since $K$ in its action on $S^{d n-1}$ is contained in the orthogonal group $O(d n)$, we have that $\mathcal{H}^{k}$, the spherical harmonics of degree $k$, is invariant under the action of $K$. Since $L^{2}\left(S^{d n-1}\right) \sim \sum_{k=0}^{\infty} \mathcal{H}^{k}$ the problem of decomposing $L^{2}\left(S^{d n-1}\right)$ according to the action of $K$ reduces to the problem of decomposing each $\mathcal{F}^{k}$ according to the action of $K$.

In this section we will decompose $\mathcal{F}^{k}$ under the action of $K$ in two ways. We shall express the members of $\mathcal{H}^{k}$ as polynomials and as solutions to certain "hypergeometric type" differential equations.

Observe that the action of $K$ extends differentiably to all of $\mathrm{F}^{n}$. Let $D^{K}\left(\mathrm{~F}^{n}\right)$ denote the ring of differential operators on $\mathbf{F}^{n}$ which commute with the left action of $K$. Since our decomposition of $L^{2}(K / M)$ will depend on obtaining "enough" differential operators which commute with the left action of $K$, an explicit description of some of the members of $D^{K}\left(\mathrm{~F}^{n}\right)$ is important.

If $\mathbf{F}=\mathbf{R}$ let $x_{1}, \ldots, x_{n}$ be the standard coordinates on $\mathbf{R}^{n}$.

If $\mathbf{F}=\mathbf{C}$ let $z_{1}, \ldots, z_{n}$ be the standard coordinates on $\mathbf{C}^{n}$.

If $\mathbf{F}=\mathbf{H}$ let $w_{1}, \ldots, w_{n}$ be the standard coordinates on $\mathbf{H}^{n}$ where $w_{a}=z_{a}$ $+j z_{n+a}$ with $z_{a}, z_{n+a} \in \mathbf{C}$. (We will have occasion to use these coordinates in §6.)

Now $\mathbf{F}^{n} \cong \mathbf{R}^{d n}$ as vector spaces over $\mathbf{R}$ and $K$ is contained in $S O(d n)$. We thus have that $E=\sum_{j=1}^{d n} x_{j} \partial / \partial x_{j}$ and $\Delta=\sum_{j=1}^{d n} \partial^{2} / \partial x_{j}^{2}$ are in $D^{K}\left(\mathbf{F}^{n}\right)$ where $E$ is the Euler operator and $\Delta$ is the Laplace operator. We now have:

LEMMA 3.1. (1) If $\mathbf{F}=\mathbf{R}, E$ and $\Delta$ are in $D^{K}\left(\mathbf{R}^{n}\right)$.

(2) If $\mathbf{F}=\mathbf{C}, E, \Delta, \sum_{j=1}^{n} z_{j} \partial / \partial z_{j}$ and $\sum_{j=1}^{n} \bar{z}_{j} \partial / \partial \bar{z}_{j}$ are in $D^{K}\left(\mathbf{C}^{n}\right)$.

(3) If $\mathbf{F}=\mathbf{H}, E, \Delta$ and $\Gamma=(\bar{D}-D)^{2}-2 D_{1} \bar{D}_{1}-2 \bar{D}_{1} D_{1}$ are in $D^{K}\left(\mathbf{H}^{n}\right)$ where

$$
\begin{aligned}
D & =\sum_{a=1}^{2 n} z_{a} \frac{\partial}{\partial z_{a}}, \quad \bar{D}=\sum_{a=1}^{2 n} \bar{z}_{a} \frac{\partial}{\partial \bar{z}_{a}}, \\
D_{1} & =\sum_{a=1}^{n}\left\{\bar{z}_{a} \frac{\partial}{\partial \bar{z}_{n+a}}-z_{n+a} \frac{\partial}{\partial \bar{z}_{a}}\right\},
\end{aligned}
$$

and

$$
\bar{D}_{1}=\sum_{a=1}^{n}\left\{z_{a} \frac{\partial}{\partial \bar{z}_{n+a}}-z_{n+a} \frac{\partial}{\partial \bar{z}_{a}}\right\} .
$$

Proof. (1) and (2) are well known. To prove (3) we set

$$
\hat{I}=\left(\begin{array}{c|c|c}
i & 0 & 0 \\
\hline 0 & 0 & 0 \\
\hline 0 & 0 & -i
\end{array}\right), \quad \hat{J}=\left(\begin{array}{c|c|c}
j & 0 & 0 \\
\hline 0 & 0 & 0 \\
\hline 0 & 0 & -j
\end{array}\right), \quad \text { and } \hat{K}=\left(\begin{array}{c|c|c}
k & 0 & 0 \\
\hline 0 & 0 & 0 \\
\hline 0 & 0 & -k
\end{array}\right) \text {. }
$$

If $x \in \mathfrak{f}$ and $f: K \rightarrow \mathbf{C}$ set 


$$
(\rho(x) f)(y)=\left.\frac{d}{d t} f(y \exp t x)\right|_{t=0} .
$$

Since $\hat{I}^{2}+\hat{J}^{2}+\hat{K}^{2}$ is ad $M$-invariant it is clear that $\rho(\hat{I})^{2}+\rho(\hat{J})^{2}+\rho(\hat{K})^{2}$ is in $D^{K}\left(\mathbf{H}^{n}\right)$ and a simple calculation shows that

$$
\left(-\frac{1}{4}\right)\left(\rho(\hat{I})^{2}+\rho(\hat{J})^{2}+\rho(\hat{K})^{2}\right)=(\bar{D}-D)^{2}-2 D_{1} \bar{D}_{1}-2 \bar{D}_{1} D_{1}=\Gamma .
$$

Thus the lemma is proved.

REMARK. Observe that $\Delta E=E \Delta+2 \Delta$. We thus have that, although $E$ and $\Delta$ do not commute, $E: \mathcal{H} \rightarrow \mathcal{K}$ where $\mathcal{H}=\sum_{k=0}^{\infty} \mathcal{F}^{k}$.

The following lemma of Vilenkin [18, pp. 499-501], will be one of our most important tools in determining the decomposition of $\mathcal{F C}^{k}$ under the action of $K$.

LEMMA 3.2 (VILENKIN). Consider the following differential equation on $[0, \pi / 2]$,

$$
\begin{aligned}
\frac{1}{\cos ^{p} \varphi \sin ^{q} \varphi} \frac{d}{d \varphi} \cos ^{p} \varphi \sin ^{q} \varphi \frac{d u}{d \varphi} \\
-\left[\frac{r(r+p-1)}{\cos ^{2} \varphi}-l(l+p+q)\right] u=0 .
\end{aligned}
$$

(i) If $p=0, q \neq 0$ and $u$ is a continuous solution to (1) then l is a nonnegative integer and $u$ is a scalar multiple of

$$
(\cos \varphi)^{l} F\left(-\frac{l}{2}, \frac{1-l}{2} ; \frac{q+1}{2} ;-\tan ^{2} \varphi\right) .
$$

(ii) If $p \neq 0, q \neq 0$ and $u$ is a continuous solution to (1) then $l-v$ must be a nonnegative even integer and $u$ is a scalar multiple of

$$
(\cos \varphi)^{l} F\left(\frac{r-l}{2}, \frac{1-p-l-r}{2} ; \frac{q+1}{2} ;-\tan ^{2} \varphi\right) .
$$

Here $F(\alpha, \beta ; \gamma ; z)$ denotes the standard hypergeometric function of type $(2,1)$ (see [19]).

We review some facts concerning spherical harmonics. Let $x_{1}, \ldots, x_{n}$ be the standard coordinates for $\mathbf{R}^{n}$. Let $r^{2}=\sum_{i=1}^{n} x_{i}^{2}$ and $\Delta_{n}$ be the standard Laplacian. Let $\mathrm{PP}^{k}$ be the space of all homogeneous polynomials of degree $k$ in the variables $x_{1}, \ldots, x_{n}$ and set $\mathcal{F}^{k}=\left.\operatorname{Ker} \Delta_{n}\right|_{\mathscr{G}^{k}}$. Clearly, $\mathcal{F}^{k}$ is invariant under the action of $O(n)$. Moreover, if $\varphi \in \mathcal{H}^{k}$,

$$
\Delta_{n}\left(\varphi r^{2 t}\right)=2 t(2 k+m+2 t-2) \varphi r^{2 t-2} .
$$

Thus we obtain easily that $\mathcal{\rho}^{k-2} r^{2} \oplus \mathcal{H}^{k}=\mathcal{\rho}^{k}$.

Thus we obtain the result that $L^{2}\left(S^{n-1}\right) \sim \sum_{k=0}^{\infty} \mathcal{Y}^{k}$ where we are identifying elements of $\mathcal{H}^{k}$ and their restrictions to the unit sphere in $\mathbf{R}^{n}$. Set 
$\mathscr{H}=\sum_{k=0}^{\infty} \mathcal{H}^{k}$ and $\mathcal{P}=\sum_{k=0}^{\infty} \mathcal{P}^{k}$. Then $\mathcal{P}=\mathcal{H} \oplus \mathscr{P} r^{2}$. Let $P: \mathscr{P} \rightarrow \mathcal{H}$ be a projection on $\mathscr{H}$ with kernel $\mathscr{P} r^{2}$.

We can now state our main result of this section.

THEOREM 3.1. (1) (Kostant [10]). As a representation of $K$,

$$
L^{2}\left(S^{d n-1}\right)=\sum_{\gamma \in \hat{K}_{0}} V_{\gamma}
$$

where $K_{0}=\left\{\gamma \in \hat{K} \mid V_{\gamma}^{M} \neq(0)\right\}$. That is if $\left(\pi_{\gamma}, V_{\gamma}\right) \in \gamma \in \hat{K}_{0}, n_{\gamma}=\operatorname{dim} V_{\gamma}^{M}$ $=1$.

(2) If $\mathbf{F}=\mathbf{R}, \mathcal{H}^{k}$ is irreducible under the action of $K$ for $n \geqslant 3$. Moreover,

$$
\left(\mathcal{T C}^{k}\right)^{M}=\mathrm{CP}\left(x_{1}^{k}\right)=\mathrm{Cr} r^{k} \cos ^{k} \xi F\left(-\frac{k}{2}, \frac{1-k}{2} ; \frac{n-1}{2} ;-\tan ^{2} \xi\right)
$$

where $x_{1}=r \cos \xi$, and $x_{i}=r \sin \xi \omega_{i}(i \geqslant 2)$ with $\sum_{i=2}^{n} \omega_{i}^{2}=1$ and $0<\xi<$ $\pi$.

(3) If $\mathbf{F}=\mathbf{C}$ let $\mathscr{P}^{p, q}=\left\{f \in \mathscr{P}^{p+q} \mid f\left(\alpha z_{1}, \ldots, \alpha z_{n}\right)=\alpha^{p} \bar{\alpha}^{q} f\left(z_{1}, \ldots, z_{n}\right)\right.$ for all $\alpha \in \mathrm{C}\}$. Let $\mathcal{F}^{p, q}=\mathcal{F}^{p+q} \cap \mathcal{P}^{p, q}$. Then $L^{2}\left(S^{2 n-1}\right) \sim \Sigma_{p, q>0} \mathcal{F C}^{p, q}$ and each $\mathcal{S C}^{p, q}$ is irreducible under the action of $K$. Furthermore,

$$
\left(\mathcal{F}^{(p, q}\right)^{M}=\mathbf{C P}\left(z_{1}^{p} \bar{z}_{1}^{q}\right)=\mathbf{C r} r^{p+q} e^{i(p-q) \varphi} \cos ^{p+q} \xi F\left(-p,-q ; n-1 ;-\tan ^{2} \xi\right)
$$

where $z_{1}=r \cos \xi e^{i \varphi}$ and $z_{i}=r \sin \xi \omega_{i}$ for $i>2$ with $\sum_{i=2}^{n}\left|\omega_{i}\right|^{2}=1,0<\varphi<$ $2 \pi$ and $0<\xi<\pi / 2$.

(4) If $\mathbf{F}=\mathbf{H}$ we use the coordinates for $\mathbf{H}^{n}$ which we have already considered. Set $r_{1}=z_{1}+\bar{z}_{1}$ and $r_{2}^{2}=\left|z_{1}\right|^{2}+\left|z_{n+1}\right|^{2}$ and let

$$
\psi_{k, k-2 j}=P\left(\sum_{i=j}^{[k / 2]}(-1)^{i}\left(\begin{array}{c}
k-i \\
i
\end{array}\right) r_{1}^{k-2 i} r_{2}^{2 i}\right) .
$$

(Note that $\Delta_{4 n}\left(\sum_{i=0}^{k / 2]}(-1)^{i}\left({ }_{i}^{k-i}\right) r_{1}^{k-2 i} r_{2}^{2 i}\right)=0$.) Let $V^{p, q}$ be the $K$-cyclic space for $\psi_{p, q}$ for $p>q$ and $p-q$ even. Then $L^{2}\left(S^{4 n-1}\right) \sim\left\{\Sigma V^{p, q} \mid p>q\right.$ and $p-q$ is even . Furthermore,

$$
\begin{aligned}
&\left(V^{p, q}\right)^{M}=\mathrm{C} \psi_{p, q} \\
&=\mathrm{C}\left[r^{p} \frac{\sin (q+1) t}{\sin t} \cos ^{P} \xi\right. \\
&\left.\qquad \cdot F\left(\frac{-p-q}{2}, \frac{-r-q-2}{2} ; 2(n-1) ;-\tan ^{2} \xi\right)\right]
\end{aligned}
$$

where $r^{2}=\sum_{i=1}^{n}\left|w_{i}\right|^{2},\left|w_{1}\right|=r \cos \xi$, and $\operatorname{Re} w_{1}=r \cos \xi \cos t$ with $0<\xi<$ $\pi / 2$ and $0<t<\pi$.

Proof. Observe that (2), (3) and (4) combined imply (1). We will therefore prove (2), (3) and (4). 
Proof of (2). Let $\nabla^{2}=x_{2}^{2}+\cdots+x_{n}^{2}$. If $f \in \mathcal{F C}^{k}$ and $f$ is invariant under the action of $M=S O(n-1), f=\sum_{j+2 l=k} a_{j, 2 l} x_{1}^{j} \nabla^{2 l}$. Since $\Delta f=0$ we have that

$$
0=\Delta f=\sum_{j+2 l=k} j(j-1) a_{j, 2 l} x_{1}^{j-2} \nabla^{2 l}+2 l(2 l+n-3) a_{j, 2 l} x_{1}^{j} \nabla^{2 l-2} .
$$

Thus we have the recursion formula

$$
(j+2)(j+1) a_{j+2,2 l}+(2 l+2)(2 l+n-1) a_{j, 2 l+2}=0
$$

where $j+2+2 l=k$. Thus knowing $a_{k, 0}$ we can determine all $a_{j, 2 l}$ uniquely. Therefore, $\left(\mathcal{F C}^{k}\right)^{M}=\mathbf{C P}\left(x_{1}^{k}\right)$.

We now use the angular coordinates of (2) and suppose that $f$ is $M$-invariant. Then $f$ is a function of only $r$ and $\xi$.

Since $d x_{1}^{2}+\cdots+d x_{n}^{2}=d r^{2}+r^{2} d \xi^{2}+r^{2} \sin ^{2} \xi\left(d \omega_{2}^{2}+\cdots+d \omega_{n}^{2}\right)$, it follows from Helgason [5, p. 387] that

$$
\Delta f=\frac{1}{r^{n-1}} \frac{\partial}{\partial r} r^{n-1} \frac{\partial}{\partial r} f+\frac{1}{r^{2}} \frac{1}{\sin ^{n-2} \xi} \frac{\partial}{\partial \xi} \sin ^{n-2} \xi \frac{\partial}{\partial \xi} f .
$$

Suppose $f \in \mathcal{F}^{k}$. Then $f(r, \xi)=r^{k} h(\xi)$. Since

$$
\frac{1}{r^{n-1}} \frac{\partial}{\partial r} r^{n-1} \frac{\partial}{\partial r} f=\frac{k(k+n-2)}{r^{2}} f
$$

we have that

$$
\frac{1}{\sin ^{n-2} \xi} \frac{d}{d \xi} \sin ^{n-2} \xi \frac{d}{d \xi} h+k(k+n-2) h=0 .
$$

(2) now follows from Lemma 3.2(i).

Proof of (3). The fact that SCp $^{p, q}$ is invariant under the action of $K$ is well known.

If $f$ is an $M$-invariant polynomial, it is a polynomial in the variables $z_{1}, \bar{z}_{1}$ and $\nabla^{2}=\left|z_{2}\right|^{2}+\cdots+\left|z_{n}\right|^{2}$. So if $f \in \mathcal{H}^{p, q}, f=\Sigma a_{j, k, 2 l} z_{1}^{j} \bar{z}_{1}^{k} \nabla^{2 l}$ where $j+k$ $+2 l=p+q$ and $j-k=p-q$. Now

$$
\begin{aligned}
0=\Delta f= & \sum 4 j k a_{j, k, 2 l} z_{1}^{j-1} \bar{z}_{1}^{k-1} \nabla^{2 l} \\
& +\sum 2 l(2 l+2 n-4) a_{j, k, 2 l} z_{1}^{j} \bar{z}_{1}^{k} \nabla^{2 l-2} .
\end{aligned}
$$

Thus we have the recursion formula

$$
4(j+1)(k+1) a_{j+1, k+1,2 l}+(2 l+2)(2 l+2 n-2) a_{j, k, 2 l+2}=0 .
$$

Thus if we know $a_{p, q, 0}$ we can determine all $a_{j, k, 2 l}$ uniquely. Thus we have $\left(\mathcal{F}^{p, q}\right)^{M}=\mathrm{CP}\left(z_{1}^{p} \bar{z}_{1}^{p q, q,}\right)$.

Using the angular coordinates of (3) we have that if $f$ is $M$-invariant, $f$ is a function of only $r, \xi$ and $\varphi$. Again using Helgason [5, p. 387], we have 


$$
\begin{aligned}
\Delta f= & \frac{1}{r^{2 n-1}} \frac{\partial}{\partial r} r^{2 n-1} \frac{\partial}{\partial r} f \\
& +\frac{1}{r^{2} \cos \xi \sin ^{2 n-3} \xi} \frac{\partial}{\partial \xi} \cos \xi \sin ^{2 n-3} \xi \frac{\partial}{\partial \xi} f \\
& +\frac{1}{r^{2} \cos ^{2} \xi} \frac{\partial^{2}}{\partial \varphi^{2}} f .
\end{aligned}
$$
and

If $f \in \mathcal{F}\left(p, q, f(r, \xi, \varphi)=r^{p+q} e^{i(p-q) \varphi} h(\xi)\right.$ and thus $\left(\partial^{2} f / \partial \varphi^{2}\right)=-(p-q)^{2} f$

$$
\frac{1}{r^{2 n-1}} \frac{\partial}{\partial r} r^{2 n-1} \frac{\partial}{\partial r} f=(p+q)(p+q+2 n-2) f .
$$

Since $\Delta f=0$,

$$
\begin{aligned}
\frac{1}{\cos \xi \sin ^{2 n-3} \xi} & \frac{\partial}{\partial \xi} \cos \xi \sin ^{2 n-3} \xi \frac{\partial}{\partial \xi} h \\
& +\left(\frac{-(p-q)^{2}}{\cos ^{2} \xi}+(p+q)(p+q+2 n-2)\right) h=0 .
\end{aligned}
$$

(3) now follows from Lemma 3.2.

Proof of (4). Recall that $\mathscr{P}=\mathcal{H} \oplus r^{2} \mathcal{P}$ and note that $\left(\mathscr{P}^{k}\right)^{M}=$ $\sum_{p+2 q+2 t=k} \mathrm{Cr}_{1}^{p} r_{2}^{2 q} r^{2 t}$.

Since $\Gamma$ is in the enveloping algebra of $\mathfrak{f}, \Gamma$ and $\Delta$ commute. A simple calculation shows that

$$
\operatorname{Tr}_{1}^{p} r_{2}^{2 q}=p(p+2) r_{1}^{p} r_{2}^{2 q}-4 p(p-1) r_{1}^{p-2} r_{2}^{2 q+2}
$$

Thus if $\Sigma a_{k, j} r_{1}^{k-2 j} r_{2}^{2 j}=f, \Gamma f=\lambda f, a_{k, i}=0$ for $i<s$, and $a_{k, s} \neq 0, \lambda=$ $(k-2 s)(k-2 s+2)$,

$$
(j-s)(k-(s+j)+1) a_{k, j}=-(k-2 j+2)(k-2 j+1) a_{k, j-1} \text {. }
$$

So $a_{k, i+s}=(-1)^{i}\left(\begin{array}{c}k-2 s-i \\ i\end{array}\right) a_{k, s}$.

We have thus shown that the only eigenvalues of $\Gamma$ in $\left(\rho^{k}\right)^{M} \bmod \left(\rho^{k}\right)^{M} \cap$ $\left(r^{2} \mathscr{P}\right)^{M}$ are $(k-2 s)(k-2 s+2)$ and each appears with multiplicity one. This clearly proves that $\left(V^{p, q}\right)^{M}=\mathbf{C} \psi_{p, q}$. We now consider the coordinates $r^{2}=\sum_{i=1}^{n}\left|w_{i}\right|^{2},\left|w_{1}\right|=r \cos \xi$ where $0<\xi<\pi / 2, w_{i}=r \sin \xi q_{i}$ for $i>2$ with $\sum_{i=2}^{n}\left|q_{i}\right|^{2}=1$ and

$$
w_{1}=r \cos \xi(\cos t+(\sin t) y)
$$

where $0<t<\pi$ and $y \in \mathbf{F}, \operatorname{Re} y=0$ and $|y|^{2}=1$.

If $f$ is $M$-invariant $f$ depends only on $r, \xi$ and $t$ and 


$$
\begin{aligned}
\Delta f= & \frac{1}{r^{4 n-1}} \frac{\partial}{\partial r} r^{4 n-1} \frac{\partial f}{\partial r} \\
& +\frac{1}{r^{2}} \frac{1}{(\cos \xi)^{3}(\sin \xi)^{4 n-5}} \frac{\partial}{\partial \xi} f \\
& +\frac{1}{r^{2}} \frac{1}{(\cos \xi)^{2}}\left[\frac{1}{\sin ^{2} t} \frac{\partial}{\partial t} \sin ^{2} t \frac{\partial}{\partial t}\right] f
\end{aligned}
$$

Noting that $-\Gamma f=\left(1 / \sin ^{2} t\right)\left(\partial \sin ^{2} t / \partial t\right)(\partial f / \partial t)$ we have that if $f \in V^{p, q}$, $-\Gamma f=-q(q+2) f$. Thus we see that $f(r, \xi, t)=r^{p}((\sin (q+1) t) / \sin t) h(\xi)$ and $h(\xi)$ satisfies the differential equation

$$
\begin{aligned}
\frac{1}{(\cos \xi)^{3}(\sin \xi)^{4 n-5}} & \frac{d}{d \xi}(\cos \xi)^{3}(\sin \xi)^{4 n-5} \frac{d}{d \xi} h \\
& +\frac{-q(q+2)}{\cos ^{2} \xi}+p(p+4 n-2) h=0
\end{aligned}
$$

(4) now follows from Lemma 3.2.

This completes the proof of Theorem 3.1.

REMARKS. If $\mathbf{F}=\mathbf{R}$ set $e_{m}=\cos ^{m} \xi F(-m / 2,(1-m) / 2 ;(n-1) / 2$; $\left.-\tan ^{2} \xi\right)$. If $\mathbf{F}=\mathbf{C}$ set

$$
e_{p+q, p-q}=e^{i(p-q) \varphi} \cos ^{p+q} \xi F\left(-p,-q ; n-1 ;-\tan ^{2} \xi\right) .
$$

This of course provides another parametrization for the $K$-irreducible spaces of harmonics when $\mathbf{F}=\mathbf{C}$. If $\mathbf{F}=\mathbf{H}$ set

$$
e_{m, l}=\frac{\sin (l+1) t}{\sin t} \cos ^{m} \xi F\left(\frac{-m-l}{2}, \frac{-m-l-2}{2} ; 2(n-1) ;-\tan ^{2} \xi\right) .
$$

In $\S 8$, we will compute characters and we will use the classification of the spaces $V^{p, q}$ given in Theorem 3.1(4) in terms of highest weights on $S p(n) \times$ $S p(1)$.

The Dynkin diagram for $S p(n) \times S p(1)$ is

$$
\begin{array}{ccccc}
\alpha_{1} & \alpha_{2} & \cdots & 0 & 0
\end{array}
$$

where the $\alpha_{i}$ 's are the simple roots. Let $\lambda_{i}$ be the basic weight such that $2\left\langle\lambda_{i}, \alpha_{j}\right\rangle /\left\langle\alpha_{j}, \alpha_{j}\right\rangle=\delta_{i j}$.

LEMMA 3.3. Suppose that relative to a choice of Weyl chamber in $S p(n) \times$ $S p(1) \mathcal{H}^{1}$ has highest weight $\mu$ and $\mathcal{H}^{2}=V^{2,2} \oplus V^{2,0}$ with $V^{2,2}$ having highest weight $2 \mu$ and $V^{2,0}$ having highest weight $\nu$. Then $\mu$ and $\nu$ are independent over $\mathbf{R}$ and $\mathcal{H}^{k}$ has precisely the highest weights $m_{1} \mu+m_{2} \nu$ with $m_{1}+2 m_{2}=k$, 
$m_{i} \geqslant 0, m_{i}$ an integer. $V^{p, q}$ has highest weight $q\left(\lambda_{1}+\lambda_{n+1}\right)+(p-q) / 2 \lambda_{n}$.

PROOF. Since every representation of $S p(n) \times S p(1)$ is self-dual, the representation of $S p(n) \times S p(1)$ on $\mathbf{H}^{n}$ is the same as the representation of $S p(n) \times S p(1)$ on $\mathcal{H}^{1}=V^{1,1}$. Thus $\mu=\lambda_{1}+\lambda_{n+1}$.

Now $\left(V^{2,0}\right)^{M}$, in fact, is fixed under the group $K_{0}=S p(1) \times S p(n-1) \times$ $S p(1)$. Thus $\nu=m \lambda_{n}$ for some positive integer $m$ since $S p(n) \times S p(1) / K_{0}$ is a symmetric space. By the Weyl dimension formula we have $m=1$. Thus we have that $\mu$ and $\nu$ are independent over $\mathbf{R}$.

Suppose the highest weights on $\mathcal{H}^{l}$ are precisely $m_{1} \mu+m_{2} \nu$ with $m_{1}+2 m_{2}$ $=l$ and the $m_{i}$ nonnegative integers for $1<l<k$. We now prove the result for $\mathcal{H}^{k}$. As $\mathcal{P}^{k} \cong \mathcal{H}^{k} \oplus \mathcal{P}^{k-2} r^{2}$ as a representation of $S p(n) \times S p(1)$ we have that highest weights of the form $m_{1} \mu+m_{2} \nu$ with $m_{1}+2 m_{2}=k$ cannot occur in $\mathfrak{P}^{k-2}$. If $m_{1}+2 m_{2}=k$ we see that $S^{m_{1}}\left(V^{1,1}\right) \otimes S^{m_{2}}\left(V^{2, g}\right)$ is a subrepresentation of $S p(n) \times S p(1)$ in $\rho^{k}$ and contains the highest weight $m_{1} \mu+m_{2} \nu$. As $\mathcal{F}^{k}$ contains [k/2] irreducible subrepresentations of $S p(n) \times$ $S p(1)$ and there are $[k / 2]$ weights of the form $m_{1} \mu+m_{2} \nu$ with $m_{1}, m_{2}$ nonnegative integers such that $m_{1}+2 m_{2}=k$ we are done.

It is now a trivial matter by examining the formula for $\left(V^{p, q}\right)^{M}$ that the highest weight on $V^{p, q}$ is $q \mu+(p-q) / 2 \nu$.

4. The action of $a$ on $X^{\nu}$. Let $H=e_{1} \otimes e_{n+1}^{*}+e_{n+1} \otimes e_{1}^{*}$. Since $\operatorname{dim} a=1$ the map $a_{\mathbf{C}}^{*} \rightarrow \mathbf{C}$ given by $\nu \rightarrow \nu(H)$ is an isomorphism and we shall abuse notation by identifying $\nu$ with $\nu(H)$. Using this identification we have that

$$
\left(\pi_{\nu}(g) f\right)(b)=\left[\frac{1-\|g \cdot 0\|^{2}}{(1-\langle g \cdot 0, b\rangle)^{2}}\right)^{\nu / 2} f\left(g^{-1} b\right)
$$

where $g \in G, b \in S^{d n-1}$ and $f \in H^{\nu}$. Moreover if we define

$$
1_{\nu}\left(g^{-1} b\right)=\left(\left(1-\|g \cdot 0\|^{2}\right) /|1-\langle g \cdot 0, b\rangle|^{2}\right)^{\nu / 2}
$$

we have that $H^{\nu}=1_{\nu} \cdot H^{0}$ where the product has the obvious meaning.

In $\$ 3$ we found an explicit formula for the $M$-fixed vectors of $X^{\nu}$. Since Ad $M(H)=H$ it is clear that $\pi_{\nu}(H)\left(X^{\nu}\right)^{M} \subset\left(X^{\nu}\right)^{M}$. In this section we will find explicit formulas for the restriction of $\pi_{\nu}(H)$ to $\left(X^{\nu}\right)^{M}$.

Suppose $f \in H^{0}$ and $y=\left(y_{1}, \ldots, y_{n}\right) \in S^{d n-1}$. A simple calculation yields

$$
\pi_{\nu}(H) 1_{\nu} f(y)=(\nu / 2)\left(y_{1}+\bar{y}_{1}\right)\left(1_{\nu} f\right)(y)+1_{\nu}(y) \pi_{0}(H) f(y) .
$$

Since $1_{\nu}(b)=1$ for all $b$ in $S^{d n-1}$ we will abuse notation and write $f$ and $1_{\nu} f$. In order to compute explicitly $\pi_{\nu}(H)$ restricted to $\left(X^{\nu}\right)^{M}$, we first state some easily derived facts concerning the hypergeometric function $F(\alpha, \beta ; \gamma ; z)$. 
LEMMA 4.1.

(1) $(d / d z) F(\alpha, \beta ; \gamma ; z)=(\alpha \beta / \gamma) F(\alpha+1, \beta+1 ; \gamma+1 ; z)$.

(2)

$$
\begin{aligned}
(\gamma-\beta-\alpha) F(\alpha, \beta ; \gamma ; z)= & (\gamma-\beta) F(\alpha, \beta-1 ; \gamma ; z) \\
& -\alpha(1-z) F(\alpha+1, \beta ; \gamma ; z) .
\end{aligned}
$$

$$
\begin{aligned}
(\gamma-\beta-\alpha) F(\alpha, \beta ; \gamma ; z)= & (\gamma-\alpha) F(\alpha-1, \beta ; \gamma ; z) \\
& -\beta(1-z) F(\alpha, \beta+1 ; \gamma ; z) .
\end{aligned}
$$

(3)

$$
\begin{aligned}
& F(\alpha, \beta+1 ; \gamma ; z)- F(\alpha, \beta ; \gamma ; z) \\
&=(\alpha z / \gamma) F(\alpha+1, \beta+1 ; \gamma+1 ; z) . \\
& F(\alpha+1, \beta ; \gamma ; z)-F(\alpha, \beta ; \gamma ; z) \\
&=(\beta z / \gamma) F(\alpha+1, \beta+1 ; \gamma+1 ; z) .
\end{aligned}
$$

THEOREM 4.1. (1) If $\mathbf{F}=\mathbf{R}$,

$$
\begin{aligned}
\pi_{\nu}(H) e_{m}=\frac{1}{n+2 m-1}\left[(n+2 m-2)(\nu+m) e_{m+1}\right. & \\
& \left.+m(\nu-m-n+2) e_{m-1}\right] .
\end{aligned}
$$

(2) If $\mathbf{F}=\mathbf{C}$ or $\mathbf{F}=\mathbf{H}$,

$$
\begin{aligned}
& \pi_{\nu}(H) e_{m, l}=\frac{1}{2(d n-2+2 m)} \\
& \cdot\left[(d n-2+l+m)(\nu+l+m) e_{m+1, l+1}\right. \\
& +(m-l)(\nu-m+l-d n+2) e_{m-1, l+1} \\
& +(m-l+d n-d)(m-l+\nu-d+2) e_{m+1, l-1} \\
& \left.+(l+m+d-2)(\nu-m-l-d n-d+4) e_{m-1, l-1}\right] \text {. }
\end{aligned}
$$

Proof. Proof of (1). From equation (*) we have

$$
\pi_{\nu}(H) e_{m}(\xi)=\nu \cos \xi e_{m}(\xi)+\pi_{0}(H) e_{m}(\xi) .
$$

Differentiation by parts yields $\pi_{0}(H) e_{m}(\xi)=\sin \xi(d / d \xi) e_{m}(\xi)$. Thus,

$$
\begin{aligned}
\pi_{\nu}(H) e_{m}(\xi)= & \nu \cos ^{m+1} \xi F\left(\frac{-m}{2}, \frac{1-m}{2} ; \frac{n-1}{2} ;-\tan ^{2} \xi\right) \\
& -m \cos ^{m-1} \xi \sin ^{2} \xi F\left(\frac{-m}{2}, \frac{1-m}{2} ; \frac{n-1}{2} ;-\tan ^{2} \xi\right) \\
& +\frac{m(1-m)}{n-1} \cos ^{m-1} \xi \tan ^{2} \xi \\
& \quad \cdot F\left(\frac{-m}{2}+1, \frac{1-m}{2}+1 ; \frac{n-1}{2}+1 ;-\tan ^{2} \xi\right) .
\end{aligned}
$$


From Lemma 4.1(3')

$$
\begin{aligned}
\frac{m(1-m)}{n-1} & \cos ^{m-1} \xi \tan ^{2} \xi F\left(\frac{-m}{2}+1, \frac{1-m}{2}+1 ; \frac{n-1}{2}+1 ;-\tan ^{2} \xi\right) \\
= & -m \cos ^{m-1} \xi F\left(\frac{2-m}{2}, \frac{1-m}{2} ; \frac{n-1}{2} ;-\tan ^{2} \xi\right) \\
& +m \cos ^{m-1} \xi F\left(\frac{-m}{2}, \frac{1-m}{2} ; \frac{n-1}{2} ;-\tan ^{2} \xi\right) \\
= & -m e_{m-1}(\xi)+m \cos ^{m-1} \xi F\left(\frac{-m}{2}, \frac{1-m}{2} ; \frac{n-1}{2} ;-\tan ^{2} \xi\right)
\end{aligned}
$$

So

$$
\begin{aligned}
\pi_{\nu}(H) e_{m}(\xi)= & (\nu+m) \cos ^{m+1} \xi \\
& \cdot F\left(\frac{-m}{2}, \frac{1-m}{2} ; \frac{n-1}{2} ;-\tan ^{2} \xi\right)-m e_{m-1}(\xi) .
\end{aligned}
$$

By Lemma 4.1(1)

$$
\begin{aligned}
& (\nu+m) \cos ^{m+1} \xi F\left(\frac{-m}{2}, \frac{1-m}{2} ; \frac{n-1}{2} ;-\tan ^{2} \xi\right) \\
& \quad=(\nu+m) \frac{(n+m-2)}{(n+2 m-2)} e_{m+1}(\xi)+(\nu+m) \frac{(m)}{(n+2 m-2)} e_{m-1}(\xi) .
\end{aligned}
$$

This proves (1).

Proof of (2). (a) Suppose that $\mathbf{F}=\mathbf{C}$ and let $e_{m, l}(\xi, \varphi)=h_{m, l}(\xi) e^{i l \varphi}$. Then differentiation by parts yields

$$
\begin{aligned}
\pi_{\nu}(H) e_{m, l}(\xi, \varphi)= & \frac{\nu}{2} \cos \xi h_{m, l} \\
+ & +\frac{1}{2} \sin \xi \frac{d h_{m, l}}{d \xi} \\
& +\left[\frac{\nu}{2} \cos \xi h_{m, l}+\frac{1}{2} \sin \xi \frac{d h_{m, l}}{d \xi}\right. \\
& \left.\left.-\frac{l}{2}\left(\cos \xi+(\cos \xi)^{-1}\right) h_{m, l}\right] h_{m, l}\right] e^{i(l-1) \varphi} .
\end{aligned}
$$

Set

$$
L_{1}\left(h_{m, l}\right)=\frac{\nu}{2} \cos \xi h_{m, l}+\frac{1}{2} \sin \xi \frac{d h_{m, l}}{d \xi}+\frac{l}{2}\left(\cos \xi+(\cos \xi)^{-1}\right) h_{m, l}
$$

and 


$$
L_{2}\left(h_{m, l}\right)=\frac{\nu}{2} \cos \xi h_{m, l}+\frac{1}{2} \sin \xi \frac{d h_{m, l}}{d \xi}-\frac{l}{2}\left(\cos \xi+(\cos \xi)^{-1}\right) h_{m, l}
$$

Then

$$
\begin{aligned}
L_{1}\left(h_{m, l}\right)= & \frac{\nu+l}{2} \cos ^{m+1} \xi F\left(\frac{l-m}{2}, \frac{-l-m}{2} ; n-1 ;-\tan ^{2} \xi\right) \\
& +\frac{l}{2} \cos ^{m-1} \xi F\left(\frac{l-m}{2}, \frac{-l-m}{2} ; n-1 ;-\tan ^{2} \xi\right) \\
& -\frac{m}{2} \cos ^{m-1} \xi \sin ^{2} \xi F\left(\frac{l-m}{2}, \frac{-l-m}{2} ; n-1 ;-\tan ^{2} \xi\right) \\
& +\left(\frac{l-m}{2}\right)\left(\frac{-l-m}{2}\right)\left(\frac{1}{n-1}\right) \cos ^{m-1} \xi\left(-\tan ^{2} \xi\right) \\
& \cdot F\left(\frac{l-m}{2}+1, \frac{-l-m}{2}+1 ; n ;-\tan ^{2} \xi\right) .
\end{aligned}
$$

By Lemma 4.1(3')

$$
\begin{aligned}
\left(\frac{l-m}{2}\right)\left(\frac{-l-m}{2}\right)\left(\frac{1}{n-1}\right) \cos ^{m-1} \xi\left(-\tan ^{2} \xi\right) \\
\cdot F\left(\frac{l-m}{2}+1, \frac{-l-m}{2}+1 ; n ;-\tan ^{2} \xi\right) \\
=\left(\frac{l-m}{2}\right) h_{m-1, l+1}-\left(\frac{l-m}{2}\right) \cos ^{m-1} \xi \\
\cdot F\left(\frac{l-m}{2}, \frac{-l-m}{2} ; n-1 ;-\tan ^{2} \xi\right) .
\end{aligned}
$$

Thus,

$$
\begin{aligned}
L_{1}\left(h_{m, l}\right)= & \frac{l-m}{2} h_{m-1, l+1}+\frac{\nu+l+m}{2} \cos ^{m+1} \xi \\
& \cdot F\left(\frac{l-m}{2}, \frac{-l-m}{2} ; n-1 ;-\tan ^{2} \xi\right) .
\end{aligned}
$$

Applying (2) of Lemma 4.1 gives

$$
\begin{aligned}
& \frac{\nu+l+m}{2} \cos ^{m+1} \xi F\left(\frac{l-m}{2}, \frac{-l-m}{2} ; n-1 ;-\tan ^{2} \xi\right) \\
& =\frac{\nu+l+m}{2}\left[\left(\frac{2 n-2+l+m}{2 n-2+2 m}\right) h_{m+1, l+1}+\left(\frac{m-l}{2 n-2+2 m}\right) h_{m-1, l+1}\right] .
\end{aligned}
$$

Therefore we obtain 


$$
\begin{aligned}
{\left[L_{1}\left(h_{m, l}\right)\right] e^{i(l+1) \varphi}=\frac{1}{2(2 n-2+2 m)} } & \\
& \quad \cdot\left[(2 n-2+l+m)(\nu+l+m) e_{m+1, l+1}\right. \\
& \left.\quad+(m-l)(\nu-m+l-2 n+2) e_{m-1, l+1}\right] .
\end{aligned}
$$

Now

$$
\begin{aligned}
L_{2}\left(h_{m, l}\right)= & \frac{\nu-l}{2} \cos ^{m+1} \xi F\left(\frac{l-m}{2}, \frac{-l-m}{2} ; n-1 ;-\tan ^{2} \xi\right) \\
& +\frac{-l}{2} \cos ^{m-1} \xi F\left(\frac{l-m}{2}, \frac{-l-m}{2} ; n-1 ;-\tan ^{2} \xi\right) \\
& -\frac{m}{2} \cos ^{m-1} \xi \sin ^{2} \xi F\left(\frac{l-m}{2}, \frac{-l-m}{2} ; n-1 ;-\tan ^{2} \xi\right) \\
& +\left(\frac{l-m}{2}\right)\left(\frac{-l-m}{2}\right)\left(\frac{1}{n-1}\right)\left(-\tan ^{2} \xi\right) \cos ^{m-1} \xi \\
& \cdot F\left(\frac{l-m}{2}+1, \frac{-l-m}{2}+1 ; n ;-\tan ^{2} \xi\right) .
\end{aligned}
$$

If we apply Lemma 4.1(3) to the fourth term on the right-hand side of our equation we obtain

$$
\begin{aligned}
L_{2}\left(h_{m, l}\right)= & \frac{-l-m}{2} h_{m-1, l-1}+\frac{\nu-l+m}{2} \cos ^{m+1} \xi \\
& \cdot F\left(\frac{l-m}{2}, \frac{-l-m}{2} ; n-1 ;-\tan ^{2} \xi\right) .
\end{aligned}
$$

By Lemma 4.1(2'), $\cos ^{m+1} \xi F\left((l-m) / 2,(-l-m) / 2 ; n-1 ;-\tan ^{2} \xi\right)$ gives us

$$
\begin{aligned}
{\left[L_{2}\left(h_{m, l}\right)\right] e^{i(l-1) \varphi}=} & \frac{1}{2(2 n-2+2 m)} \\
& \cdot\left[(2 n-2+l+m)(m-l+\nu) e_{m+1, l-1}\right. \\
& \left.\quad+(l+m)(\nu-m-l-2 n+2) e_{m-1, l-1}\right] .
\end{aligned}
$$

(b) Suppose now that $\mathbf{F}=\mathbf{H}$ and let $e_{m, l}(\xi, t)=\chi_{l}(t) h_{m, l}(\xi)$. Then

$$
\begin{aligned}
\pi_{\nu}(H) e_{m, l}(\xi, t)= & \nu \cos \xi \cos t \chi_{l}(t) h_{m, l}(\xi) \\
& +\sin \xi \cos t \chi_{l}(t) \frac{d h_{m, l}(\xi)}{d \xi} \\
& +\left(\cos \xi+(\cos \xi)^{-1} \sin t \frac{d \chi_{l}(t)}{d t}\right) h_{m, l}(\xi) .
\end{aligned}
$$


As $\chi_{1}(t)=(\sin (l+1) t) / \sin t$ we have that

$$
\cos t \chi_{1}(t)=\frac{1}{2} \chi_{1+1}(t)+\frac{1}{2} \chi_{1-1}(t)
$$

and

$$
\sin t \frac{d}{d t} \chi_{l}(t)=\frac{l}{2} \chi_{1+1}(t)-\frac{l+2}{2} \chi_{l-1}(t)
$$

Thus

$$
\begin{aligned}
\pi_{\nu}(H) e_{m, l}(\xi, t)= & \frac{\nu}{2} \cos \xi h_{m, l}(\xi)+\frac{1}{2} \sin \xi \frac{d h_{m, l}}{d \xi} \\
& \left.+\frac{l}{2}\left(\cos \xi+(\cos \xi)^{-1}\right) h_{m, l}\right] \chi_{l+1}(t) \\
& +\left[\frac{\nu}{2} \cos \xi h_{m, l}(\xi)+\frac{1}{2} \sin \xi \frac{d h_{m, l}}{d \xi}\right. \\
& \left.-\frac{l+2}{2}\left(\cos \xi+(\cos \xi)^{-1}\right) h_{m, l}\right] \chi_{l-1}(t) .
\end{aligned}
$$

Our result now follows by using the argument of (a). This completes the proof of the theorem.

5. The composition series for the principal series. In this section $G$ will be a classical split rank one group. Our main goal in this section will be a complete description of the composition series which arises in $\left(\pi_{v}, X^{\nu}\right)$ when $\pi_{v}$ is reducible. For this purpose we need the following proposition.

Proposition 5.1. (1) There is a G-invariant nondegenerate sesquilinear pairing between $\left(\pi_{\nu}, H^{\nu}\right)$ and $\left(\pi_{d(n+1)-2-\bar{p}}, H^{d(n+1)-2-\bar{\nu}}\right)$.

(2) Let $f$ be an element of $V_{\gamma}$ for some $\gamma \in \hat{K}_{0}$. Then, if $1_{\gamma} f$ is a cyclic vector for $\left(\pi_{v}, H^{\nu}\right)$, the cyclic space for $1_{d(n+1)-2-i}$ is infinitesimally irreducible.

See [22] for a proof of this result.

Recall the definition of the function $e$ for $g$

$$
e(\lambda)=\Gamma\left(\frac{1}{2}\left(\frac{1}{2} m_{\alpha}+1+\left\langle i \lambda, \alpha_{0}\right\rangle\right)\right) \Gamma\left(\frac{1}{2}\left(\frac{1}{2} m_{\alpha}+m_{2 \alpha}+\left\langle i \lambda, \alpha_{0}\right\rangle\right)\right) .
$$

See [6] for an explanation of the terms.

Then $e(i(\rho-\nu \alpha))=\Gamma\left(\frac{1}{2}\left(\nu+1-\mathrm{m}_{2 \alpha}\right)\right) \Gamma\left(\frac{1}{2} \nu\right)$ where

So

$$
m_{2 \alpha}= \begin{cases}0 & \text { if } \mathbf{F}=\mathbf{R} \\ 1 & \text { if } \mathbf{F}=\mathbf{C} \\ 3 & \text { if } \mathbf{F}=\mathbf{H}\end{cases}
$$

$$
\begin{gathered}
e(i(\rho-\nu \alpha)) e(-i(\rho-\nu \alpha))=\Gamma\left(\frac{1}{2} \nu\right) \Gamma\left(-\frac{1}{2} \nu\right) \Gamma\left(\frac{1}{2}\left(\nu+1-m_{2 \alpha}\right)\right) \\
\cdot \Gamma\left(\frac{1}{2}\left(-\nu+1-m_{2 \alpha}\right)\right) .
\end{gathered}
$$


Let $X_{0}^{p}$ be the cyclic space for $1_{\nu}$ in $X^{\nu}$.

THEOREM 5.1. (1) (Kostant [10], Helgason [6]). $X_{0}^{\nu}=X^{\nu}$ if and only if $e(i(\rho-\nu \alpha)) \neq 0 . \pi_{\nu}$ is irreducible if and only if $e(i(\rho-\nu \alpha)) e(-i(\rho-\nu \alpha)) \neq$ 0.

(2) (Takahashi [17]). Let $\mathbf{F}=\mathbf{R}$ and $\nu=-k$ where $k$ is a nonnegative integer. Then $\pi_{-k}$ leaves $V_{k}=\sum_{j=0}^{k} \mathscr{F C}^{j}$ invariant, and the induced representations on $V_{k}$ and $X^{-k} / V_{k}$ are irreducible. By duality, $\pi_{n-1+k}$ leaves $W_{k}=$ $\sum_{j=k+1}^{\infty} \mathcal{F C}^{j}$ invariant, and $W_{k}$ and $X^{n-1+k} / W_{k}$ are irreducible.

(3) Let $\mathbf{F}=\mathbf{C}$ and $\nu=-2 l$ where $l$ is a nonnegative integer. Then $\pi_{-2 l}$ leaves

$$
\begin{gathered}
L_{2 l}=\sum_{p, q=0}^{l} \mathcal{F}^{p, q}, \quad H_{2 l}^{+}=\sum_{p=0}^{\infty} \sum_{q=0}^{l} \mathcal{F}^{p, q}, \\
H_{2 l}^{-}=\sum_{q=0}^{\infty} \sum_{p=0}^{l} \mathcal{F}^{p, q}, \quad H_{2 l}^{+}+H_{2 l}^{-}
\end{gathered}
$$

and $X^{-2 l}$ invariant with $L_{2 l}, H_{2 l}^{+} / L_{2 l}, H_{2 l}^{-} / L_{2 l}$ and $X^{-2 l} /\left(H_{2 l}^{+}+H_{2 l}^{-}\right)$ irreducible under the action induced by $\pi_{-2 l} \cdot \pi_{2 n+2 l}$ leaves

$$
\begin{gathered}
U_{2 l}=\sum_{p=l+1}^{\infty} \sum_{q=l+1}^{\infty} \mathcal{F}^{p, q}, \quad F_{2 l}^{+}=\sum_{p=l+1}^{\infty} \sum_{q=0}^{\infty} \mathcal{F}^{p, q}, \\
F_{2 l}^{-}=\sum_{p=0}^{\infty} \sum_{q=l+1}^{\infty} \mathcal{F}^{p, q}, \quad F_{2 l}^{+}+F_{2 l}^{-},
\end{gathered}
$$

and $X^{2 n+2 l}$ invariant and the actions induced on $U_{2 l}, F_{2 l}^{+} / U_{2 l}, F_{2 l}^{-} / U_{2 l}$, and $X^{2 n+2 l} /\left(F_{2 l}^{-}+F_{2 l}^{+}\right)$are irreducible.

(4) (i) Let $\mathbf{F}=\mathbf{H}$ and $\nu=-2 l$ where $l$ is a nonnegative integer. Then $\pi_{-2 l}$ leaves $W_{l}=\Sigma_{m+k<2 l} V^{m, k}, M_{l}=\Sigma_{m-k<2 l+2} V^{m, k}$ and $X^{-2 l}$ invariant, and $W_{l}$, $M_{l} / W_{l}$ and $X^{-2 l} / M_{l}$ are irreducible. Dualizing we have that $\tilde{W}_{l}=$ $\Sigma_{m+k>2 l} V^{m, k}, \quad \tilde{M}_{l}=\Sigma_{m-k>2 l+2} V^{m, k}$ and $X^{4 n+2+2 l}$ are invariant under $\pi_{4 n+2+2 l}$ and the induced representations on $\tilde{M}_{l}, \tilde{W}_{l} / \tilde{M}_{l}$ and $X^{4 n+2+2 l} / \tilde{W}_{l}$ are irreducible.

(ii) Again, let $\mathbf{F}=\mathbf{H}$. Then $T=\sum_{m=0}^{\infty} V^{m, m}$ and $X^{2}$ are invariant under $\pi_{2}$ and $T$ and $X^{2} / T$ are irreducible. $\tilde{T}=\Sigma_{m-k>0} V^{m, k}$ and $X^{4 n}$ are invariant under $\pi_{4 n}$ and $\tilde{T}$ and $X^{4 n} / \tilde{T}$ are irreducible.

PRoof. We will prove (1) for each case separately.

Proof of (1) and (2) for $\mathbf{F}=\mathbf{R}$. From Theorem 4.1(1) we have that $\pi_{\nu}(H)^{m} 1_{\nu} \equiv(\nu+m-1)(\nu+m-2) \cdots(\nu) e_{m} \bmod \left[1_{\nu}, e_{1}, \ldots, e_{m-1}\right]$. Thus $1_{\nu}$ is cyclic if and only if $\nu \neq-k$ where $k$ is some nonnegative integer. Since $m_{\alpha}=n-1$ and $m_{2 \alpha}=0$ this is equivalent to the condition that $e(i(\rho-\nu \alpha)) \neq 0$. By duality we have that $\pi_{\nu}$ is irreducible if and only if 
$e(-i(\rho-\bar{\nu} \alpha)) e(i(\rho-\nu \alpha)) \neq 0$. But $e(-i(\rho-\bar{\nu} \alpha)) e(i(\rho-\nu \alpha)) \neq 0$ if and only if $e(-i(\rho-\nu \alpha)) e(i(\rho-\nu \alpha)) \neq 0$. This proves (1) if $\mathbf{F}=\mathbf{R}$.

We now consider $\pi_{-k}$ and its dual representation $\pi_{n-1+k}$. From Theorem 4.1(1) we see that $1_{n-1+k}$ is a cyclic vector for $\pi_{n-1+k}$ and that the cyclic space for $1_{-k}$ is $V_{k}$. So $V_{k}$ is an irreducible subspace. From Theorem 4.1(1) we see that $e_{k+1}$ is a cyclic vector for $\pi_{-k}$ and the irreducible cyclic space for $e_{k+1}$ in $X^{n-1+k}$ is $W_{k}$. (2) now follows from Proposition 5.1(1).

Proof of (1) and (3) for F = C. Using Theorem 4.1(2) with $d=2$ we can see that 1 , will be a cyclic vector for $\pi_{\nu}$ if and only if $\nu \neq-2 k$ where $k$ is a nonnegative integer. By duality we obtain (1) when $\mathbf{F}=\mathbf{C}$.

We now consider $\pi_{-2 l}$ and its dual representation $\pi_{2 n+2 l}$ where $k$ is a nonnegative integer. Since $1_{2 n+2 l}$ is cyclic for $\pi_{2 n+2 l}$ the cyclic space for $1_{-2 l}$ in $X^{-2 l}$ is irreducible. By Theorem 4.1(2) and the remark in $\S 3$ for $\mathbf{F}=\mathbf{C}$, we see that the cyclic space for $1_{-2 l}$ is $L_{2 l}$. By further application of $4.1(2)$ and the remark in $\S 3$ for $\mathbf{F}=\mathrm{C}$ we see that $H_{2 l}^{+}, H_{2 l}^{-}, H_{2 l}^{+}+H_{2 l}^{-}$and $X^{-2 l}$ are invariant under the action of $\pi_{-2 l}$. In the same manner we see that $\pi_{2 n+2 l}$ leaves $U_{2 l}, F_{2 l}^{+}, F_{2 l}^{-}, F_{2 l}^{+}+F_{2 l}^{-}$, and $X^{2 n+2 l}$ invariant.

Observe by Theorem 4.1(2) that $e_{2 l+2,0}$ is cyclic for $\pi_{-2 l}$ and that the cyclic space for $e_{2 l+2,0}$ in $\pi_{2 n+2 l}$ is $U_{2 l}$.

By observing that the actions of $\pi_{-2 l}$ on $\mathrm{H}_{2 l}^{+}+\mathrm{H}_{2 l}^{-}$and $\pi_{-2 l}$ on $\mathrm{H}_{2 l}^{+}+$ $H_{2 l}-$ and $\pi_{2 n+2 l}$ on $X^{2 n+2 l} / U_{2 l}$ are dual (3) follows by a modification of Proposition 5.1(2).

Proof of (1) and (4) for $\mathbf{F}=\mathbf{H}$. By using Thoerem 4.1(2) $(d=4)$ we see that 1, is cyclic if and only if $\nu \neq-2 l$ where $l$ is a nonnegative integer and $\nu \neq 2$. By duality (1) now follows immediately for $\mathbf{F}=\mathbf{H}$.

(i) We consider $\pi_{-2 l}$ and its dual representation $\pi_{4 n+2+2 l}$ for $l$ a nonnegative integer. By Theorem 4.1(2) we see that $W_{l}, M_{l}$ and $X^{-2 l}$ are invariant under the action of $\pi_{-2 l}$. Similarly we see that $\tilde{W}_{l}, \tilde{M}_{l}$ and $X^{4 n+2+2 l}$ are invariant under the action of $\pi_{4 n+2+2 l}$. Also Theorem 4.1(2) tells us that $1_{4 n+2+2 l}$ is cyclic for $\pi_{4 n+2+2 l}$ and $e_{2 l+4,0}$ is cyclic for $\pi_{-2 l}$. The cyclic space for $1_{-2 l}$ is $W_{l}$ and the cyclic space for $e_{2 l+4,0}$ in $X^{4 n+2+2 l}$ is $\tilde{M}_{l}$. Now the induced action of $\pi_{4 n+2+2 l}$ on $X^{4 n+2+2 l} / \tilde{M}_{l}$ is dual to the action of $\pi_{-2 l}$ on $M_{l}$. By modifying Proposition 5.1(2) we obtain 4(i).

(ii) By Theorem 4.1(2) we have that $1_{4 n}$ is a cyclic vector for $\pi_{4 n}$. Also, we see that the cyclic space for $1_{2}$ in $X^{2}$ is $T$. Again by Theorem 4.1(2) we see that $e_{2,0}$ is a cyclic vector for $\pi_{2}$ and the cyclic space for $e_{2,0}$ in $X^{4 n}$ is $\tilde{T}$. 4(ii) now follows from Proposition 5.1.

6. The intertwining operators and the complementary series for the classical split rank one groups. In this section we compute the intertwining operators and the "partial" intertwining operators between $X^{\nu}$ and $X^{\lambda}$ for $\lambda, \nu \in \mathbf{C}$ when $G$ is one of our classical split rank one groups. We actually determine 
the linear maps $A_{0}$ from $X^{\nu}$ to $X^{\lambda}$ such that $A_{0} \pi_{\nu}(k)=\pi_{\lambda}(k) A_{0}$ for all $k \in K$ and $A_{0} \pi_{\nu}(H)=\pi_{\lambda}(H) A_{0}$.

THEOREM 6.1. Suppose that $A_{0}$ intertwines $X^{\nu}$ and $X^{\lambda}$ where $\lambda=d(n+1)-$ $2-\nu$ with $A_{0} 1_{\nu}=1_{\lambda}$ :

(i) if $\mathbf{F}=\mathbf{R}$,

$$
a_{k}(\nu)=\left.A_{0}\right|_{s^{k}}=\prod_{j=1}^{k}\left(\frac{n-2-\nu+j}{\nu+j-1}\right) I
$$

(ii) if $\mathbf{F}=\mathbf{C}$,

$$
a_{p, q}(\nu)=\left.A_{0}\right|_{s p_{0.4}}=\prod_{j=1}^{p}\left(\frac{2 n-2+2 j-\nu}{2 j-2+\nu}\right) \prod_{l=1}^{q}\left(\frac{2 n-2+2 l-\nu}{2 l-2+\nu}\right) I ;
$$

or,

(iii) if $\mathbf{F}=\mathbf{H}$,

$$
a_{p, q}(\nu)=\left.A_{0}\right|_{\nu p, q}=\prod_{j=1}^{(p-q) / 2}\left(\frac{4 n-2+2 j-\nu}{2 j-4+\nu}\right) \prod_{l=1}^{(p+q) / 2}\left(\frac{4 n+2 l-\nu}{2 l-2+\nu}\right) I .
$$

Proof. (i) Now $\pi_{v}(H) a_{k}(v) e_{k}=A_{0}\left(\pi_{\lambda}(H) e_{k}\right)$. Thus by Theorem 5.1(1) we have that

$$
\begin{array}{r}
\frac{1}{n+2 k-2}\left[(n+2 k-2)(\nu+k) a_{k}(\nu) e_{k+1}+k(\nu-k-n+2) a_{k}(\nu) e_{k-1}\right] \\
=\frac{1}{n+2 k-2}\left[(n+2 k-2)(\lambda+k) a_{k+1}(\nu) e_{k+1}\right. \\
\left.+k(\lambda-k-n+2) a_{k-1}(\nu) e_{k-1}\right] .
\end{array}
$$

This gives us a recursion relation for $a_{k+1}(\nu)$ and (i) follows. (ii) and (iii) follow in the same way.

We consider the intertwining operator $B_{0}(\nu): X^{\nu} \rightarrow X^{d(n+1)-2-\nu}$ defined by $B_{0}(\nu)=e(i(\rho-\nu \alpha)) A_{0}(\nu)$.

REMARKS. If $\mathbf{F}=\mathbf{R}$,

$$
\left.B_{0}(\nu)\right|_{\mathcal{S}^{k}}=\frac{1}{\Gamma\left(\frac{1}{2} \nu\right) \Gamma\left(\frac{1}{2}(\nu+1)\right)} a_{k}(\nu)=\frac{2^{1-\nu} \pi^{1 / 2}}{\Gamma(\nu)} a_{k}(\nu)=b_{k}(\nu) .
$$

If $\mathbf{F}=\mathbf{C}$,

$$
\left.B_{0}(\nu)\right|_{S \mathcal{C}, q}=\frac{1}{\left(\Gamma\left(\frac{1}{2} \nu\right)\right)^{2}} a_{p, q}(\nu)=b_{p, q}(\nu)
$$

If $\mathbf{F}=\mathbf{H}$,

$$
\left.B_{0}(\nu)\right|_{\nu^{p, q}}=\frac{1}{\Gamma(\nu / 2) \Gamma((\nu-2) / 2)} a_{p, q}(\nu)=b_{p, q}(\nu) .
$$


We wish to determine which of the representations $\left(\pi_{\nu}, X^{\nu}\right)$ and which of the representations defined in Theorem 5.1 have invariant pre-Hilbert space structures. In other words suppose $\left(\pi_{1}, V_{1}\right)$ is one of these representations and $\left(\pi_{2}, V_{2}\right)$ is the dual representation. We wish to determine whether or nor there is an intertwining operator $B: V_{1} \rightarrow V_{2}$ such that, for all $v$ in $V_{1},\langle B v, v\rangle>0$. Observe, for example, that if $\nu=(d(n+1) / 2)-1+i \lambda$ where $\lambda$ is real, $\pi_{\nu}$ is self-dual and the identity defines the appropriate intertwining operator.

From Proposition 5.1(1) it is clear that we can only find an invariant pre-Hilbert space structure if $\nu=\bar{\nu}$ or if $\nu=d(n+1)-2-\bar{\nu}$. Since the second case has just been dealt with, we consider the case where $\nu$ is real.

Let $B_{0}(\nu)$ be as above and let $\langle$,$\rangle denote the sesquilinear pairing on$ $H^{\nu} \times H^{d(n+1)-2-\bar{\nu}}$ in Proposition 5.1(1). (If $f \in H^{\nu}, g \in H^{d(n+1)-2-\bar{\nu}}$, $\langle f, g\rangle=\int_{K / M} f(b) \overline{g(b)} d b$.) If $\nu$ is real let $(,)_{\nu}$ be the bilinear form on $H^{\nu}$ defined as follows: For $f, g$ in $H^{\nu}$ set $(f, g)_{\nu}=\left\langle f, B_{0}(\nu) g\right\rangle$.

TheOREM 6.2 (Kostant [10]). Suppose $\nu$ is real. Then the following statements are true.

(1) If $\mathbf{F}=\mathbf{R},($,$) , induces a positive definite Hermitian form on X^{\nu}$ if and only if $0<\nu<n-1$.

(2) If $\mathbf{F}=\mathbf{C},($,$) , induces a positive definite Hermitian form on X^{\nu}$ if and only if $0<\nu<2 n$.

(3) If $\mathbf{F}=\mathbf{H},($,$) , induces a positive definite Hermitian form on X^{\nu}$ if and only if $2<\nu<4 n$.

Proof. This follows from Theorem 6.1 if one observes that all the $b_{\gamma}(\nu)$ 's are nonzero and have the same sign for each $\gamma \in \hat{K}_{0}$. (Here we are identifying the elements of $\hat{K}_{0}$ with their indexing sets.)

We use the notation of Theorem 5.1. By reasoning similar to the above we easily obtain:

THEOREM 6.3.(1) (Takahashi [17]). If $\mathbf{F}=\mathbf{R}$ the kernel of $B_{0}(-k)$ is $V_{k}$ and (, ) $)_{-k}$ induces a positive definite bilinear form on $X^{-k} / V_{k} \cong W_{k}$.

(2) If $\mathbf{F}=\mathbf{C}$ the kernel of $B_{0}(-2 l)$ is $\mathrm{H}_{2 l}^{+}+\mathrm{H}_{2 l}^{-}$and (, $)_{-2 l}$ induces a positive definite bilinear form on $X^{-2 l} /\left(H_{2 l}^{+}+H_{2 l}^{-}\right) \approx U_{2 l}$.

(3) (i) If $\mathbf{F}=\mathbf{H}$ the kernel of $B_{0}(-2 l)$ is $M_{l}$ and $(,)_{-l}$ induces a positive definite bilinear form on $X^{-2 l} / M_{l} \simeq \tilde{M}_{l}$.

(ii) If $\mathbf{F}=\mathbf{H}$ the kernel of $B_{0}(2)$ is $T$ and $(,)_{2}$ induces a positive definite bilinear form on $X^{2} / T \approx \tilde{T}$. The kernel of $B_{0}(4 n)$ is $\tilde{T}$ and $(,)_{4 n}$ induces a positive definite bilinear form on $X^{4 n} / \tilde{T} \simeq T$.

Observe that if $\mathbf{F}=\mathbf{R}$ we have exhausted our considerations. However, if $\mathbf{F}=\mathbf{C}$ or $\mathbf{H}$ there are some subquotient representations for which we have 
not determined whether or not there is a positive definite Hermitian bilinear form.

If $\mathbf{F}=\mathbf{C}$ set

$$
C_{0}(\nu)=\Gamma(\nu / 2) B_{0}(\nu)
$$

and if $\mathbf{F}=\mathbf{H}$ set

$$
C_{0}(\nu)=\Gamma((\nu-2) / 2) B_{0}(\nu)
$$

If $\nu=-2 l$ let $\{f, g\}_{-2 l}=\left\langle f, C_{0}(-2 l) g\right\rangle$.

THEOREM 6.4. (1) If $\mathbf{F}=\mathbf{C}, C_{0}(-2 l)$ is defined on $H_{2 l}^{+}+H_{2 l}^{-}$and has kernel $L_{2 l} \cdot C_{0}(-2 l)$ is an isomorphism from $\left(H_{2 l}^{+}+H_{2 l}^{-}\right) / L_{2 l}$ to $\left(F_{2 l}^{+}+F_{2 l}^{-}\right) / U_{2 l}$. Moreover, $\{,\}_{-2 l}$ induces a positive definite Hermitian form on $\left(H_{2 l}^{+}+H_{2 l}^{-}\right) / L_{2 l}$ if and only if $l=0$. Thus $H_{2 l}^{+} / L_{2 l} \cong F_{2 l}^{+} / U_{2 l}$ and $H_{2 l}^{-} / L_{2 l} \cong F_{2 l}^{-} / U_{2 l}$, and only $H_{0}^{+} / L_{0} \cong F_{0}^{+} / U_{0}$ and $H_{0}^{-} / L_{0} \cong F_{0}^{-} / U_{0}$ have invariant pre-Hilbert space structures.

(2) If $\mathbf{F}=\mathbf{H}, C_{0}(-2 l)$ is defined on $M_{l}$ and has kernel $W_{l} \cdot C_{0}(-2 l)$ induces an isomorphism of $M_{l} / W_{l}$ with $\tilde{W}_{l} / \tilde{M}_{l}$ and $\{,\}_{-2 l}$ never induces a positive definite Hermitian form.

Proof. The proof of this theorem is the same as the proof of Theorem 6.2.

By Nelson's theorem [13], we obtain:

THEOREM 6.5. Consider the representations of Theorems 6.2, 6.3 and 6.4 which have positive definite invariant Hermitian forms on them. We obtain unitary representations of $G$ on their respective completions with respect to the induced norm.

7. Kostant's $P$-matrices. In this section we give the relationship between Kostant's $P$-matrices and the intertwining operators for general semisimple groups. We show how to compute the $P$-matrix in the case of split rank 1 using the results of $\S 6$ and [7].

The Poincaré-Birkhoff-Witt theorem implies that since $g=\mathfrak{a} \oplus \mathbb{n}$, $U(g)$ (the universal enveloping algebra of $g$ ) splits into a direct sum $U(g)=$ $(U(\mathfrak{g}) \mathfrak{f}+\mathfrak{n} U(\mathfrak{g})) \oplus U(\mathfrak{a})(U(\mathfrak{a})$ is the universal enveloping algebra of $a)$. Thus if $u \in U(\mathrm{~g})$ there is a unique element $P_{u} \in U(\mathrm{a})$ so that $u-P_{u} \in$ $U(\mathfrak{g}) \mathfrak{f}+\mathfrak{n} U(\mathfrak{g})$.

If $u \in U(\mathrm{~g})$ and $\nu \in \mathrm{a}^{*}$ then $\left(\pi_{\nu}(u) 1_{\nu}\right)(e)=\nu\left(P_{u}\right)$ (here $\nu$ is extended as the unique homomorphism of $U(\mathfrak{a})$ to $\mathbf{C}$ which is $\nu$ on $\mathfrak{a})$. This is easily checked since if $X \in \mathfrak{n}$ and $f \in X^{\nu}$ then $\left(\pi_{\nu}(X) f\right)(e)=0$ and $\pi_{\nu}(\mathfrak{f}) 1_{\nu}=0$.

Set $P_{u}(\nu)=\nu\left(P_{u}\right)$.

Kostant and Rallis [11] showed that there exist $K$-invariant (under ad) subspaces $H^{*}$ and $J^{*}$ (see [11] for definitions) of $U(g)$ so that

(1) $U(\mathrm{~g})=U(\mathrm{~g}) \mathfrak{f} \oplus H^{*} J^{*}$. 
(2) $J^{*}$ is contained in the centralizer of $\sharp$ in $U(\mathrm{~g})$.

(3) $H^{*}$ as a $K$-representation is equivalent with $X^{\nu}$. (As a $K$-representation, $\left(\left.\pi_{\nu}\right|_{K}, X^{\nu}\right) \equiv\left(\left.\pi_{\mu}\right|_{K}, X^{\mu}\right)$ for all $\nu, \mu \in \mathfrak{a}_{\mathbf{C}}^{*}$. Let us denote $\left(\left.\pi_{\nu}\right|_{K}, X^{\nu}\right)$ as $(\pi, X)$.)

For $\gamma \in \hat{K}$, let $E_{\gamma}=\operatorname{Hom}_{K}\left(V_{\gamma}, H^{*}\right)$ and $\hat{E}_{\gamma}=\operatorname{Hom}_{K}\left(V_{\gamma}, X\right)$.

LEMma 7.1. There is a basis $a_{1}, \ldots, a_{l(\gamma)}$ of $E_{\gamma}\left(l(\gamma)=\operatorname{dim} V_{\gamma}^{M}\right.$ (the $M$-invariants in $\left.V_{\gamma}\right)$ ) and a basis $\nu_{1}, \ldots, \nu_{l(\gamma)}$ of $V_{\gamma}^{M}$ so that $P_{a_{i}\left(\gamma_{)}\right)}(2 \rho)=\delta_{i j}$.

Proof. If we observe that $1_{2 \rho}\left(g^{-1} k\right)=P(g K, k M)$ is the Poisson kernel of $G / X$ it is easy to see that $\pi_{2 \rho}(U(\mathrm{~g})) 1_{2 \rho}=X^{2 \rho}$. Now $\pi_{2 \rho}\left(J^{*}\right) 1_{2 \rho} \subset \mathrm{Cl}_{2 \rho}$ and

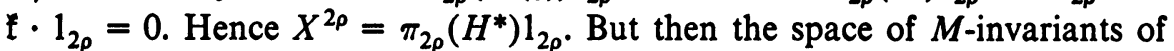
$X^{2 \rho}$ is $\sum_{\gamma \in \hat{K}^{2 \rho}} \pi_{2 \rho}\left(E_{\gamma}\left(V_{\gamma}^{M}\right)\right) 1_{2 \rho}$. Frobenius reciprocity implies that $X=$ $\Sigma_{\gamma \in \hat{K}} \psi\left(\hat{E}_{\gamma} \otimes V_{\gamma}\right)$ where

$$
\psi(A \otimes v)(k)=A\left(\tau_{\gamma}(k)^{-1} v\right) \quad\left(\rho_{\gamma}, V_{\gamma}\right) \in \gamma .
$$

Thus the $M$-invariants of $X$ are just $\Sigma_{\gamma \in \hat{K}} \psi\left(\hat{E}_{\gamma} \otimes V_{\gamma}^{M}\right) \cdot 1_{2 \rho}$. Now it is clear that $\pi_{2 \rho}\left(E_{\gamma}\left(V_{\gamma}\right)\right) 1_{2 \rho}=\psi\left(\hat{E}_{\gamma} \otimes V_{\gamma}\right)$. Thus by counting dimensions we see that there is a bijective mapping $\delta_{\gamma}: E_{\gamma} \rightarrow \hat{E}_{\gamma}$ so that

$$
\pi_{2 \rho}(a(v)) 1_{2 \rho}=\psi\left(\delta_{\gamma}(a) \otimes v\right) .
$$

The standard proof of Frobenius reciprocity implies that there is a basis $v_{1}, \ldots, v_{l(\gamma)}$ of $V_{\gamma}^{M}$ and a basis $A_{1}, \ldots, A_{l(\gamma)}$ of $\hat{E}_{\gamma}$ so that $\psi\left(A_{i} \otimes v_{j}\right)(e)=$ $\delta_{i j}$. Take $a_{i}=\delta_{\gamma}^{-1}\left(A_{i}\right)$. Q.E.D.

Definition 7.2. $P_{i j}^{\gamma}(\nu)=P_{\left.a_{i}(y)\right)}(\nu)$ for $a_{1}, \ldots, a_{l(\gamma)}, \nu_{1}, \ldots, \nu_{l(\gamma)}$ as in Lemma 7.1.

Set $P^{\gamma}(\nu)=\left(P_{i j}^{\gamma}(v)\right)$. We look at $P^{\gamma}(\nu)$ as a map of $V_{\gamma}^{M} \rightarrow V_{\gamma}^{M}$ by setting $P^{\gamma}(\nu) v_{i}=\Sigma P_{j i}^{\gamma}(\nu) v_{j}$.

If $\lambda \in\left(V_{\gamma}^{*}\right)^{M}, v \in V_{\gamma}$, define $(\lambda \otimes v)(k)=\lambda\left(\rho_{\gamma}(k)^{-1} v\right)$. Then $X_{\gamma}=\left(V_{\gamma}^{*}\right)^{M}$ $\otimes V_{\gamma}$ (the only action of $K$ is on $V_{\gamma}$ ) as a $K$-module. Define for $a \in E_{\gamma}$, $v \in V_{\gamma}^{M}, B_{\nu}^{\gamma}(a)(v)=\left(\pi_{\nu}(a(v)) \cdot 1\right)(e)$. Then $B_{\nu}^{\gamma}: E_{\gamma} \rightarrow\left(V_{\gamma}^{*}\right)^{M}$. Clearly $B_{v}^{\gamma}(a)(v)=P_{a(v)}(v)$.

Now let $T_{\nu}: H^{*} \rightarrow X$ be defined by $T_{\nu}(u)=\pi_{\nu}(u) 1_{\nu}$. Then $T_{2 \rho}$ is bijective by Lemma 7.1 .

LemMa 7.3. $T_{\nu} \circ T_{2 \rho}^{-1} \lambda \otimes v=\lambda \circ P^{\gamma}(\nu) \otimes v$, for $\lambda \in\left(V_{\gamma}^{*}\right)^{M}, v \in V_{\gamma}^{M}$.

PROOF. Let $\hat{\delta}_{\gamma}: E_{\gamma} \rightarrow\left(V_{\gamma}^{*}\right)^{M}$ be defined so that $T_{2 \rho}(a(v))=\hat{\delta}_{\gamma}(a) \otimes v$, $a \in E_{\gamma}, v \in V_{\gamma}$. By the above $B_{2 \rho}^{\gamma}(a)=\hat{\delta}_{\gamma}(a)$. Now $T_{\nu} \circ T_{2 \rho}^{-1}\left(B_{2 \rho}^{\gamma}(a) \otimes v\right)=$ $B_{\nu}^{\gamma}(a) \otimes v$. But $B_{\nu}^{\gamma}\left(a_{i}\right)\left(v_{j}\right)=P_{i j}^{\gamma}(\nu)$. Thus $B_{\nu}^{\gamma}(a)=B_{2 \rho}^{\gamma}(a) \circ P^{\gamma}(\nu)$. Hence if $B_{2 \rho}^{\gamma}(a)=\lambda$, then $T_{\nu} \circ T_{2 \rho}^{-1} \lambda \otimes v=\lambda \circ P^{\gamma}(\nu) \otimes v$.

We note that the definition of the $P^{\gamma}(\nu)$ implies

Lemma 7.4. $\pi_{\nu}(U(\mathrm{~g})) 1_{\nu}=X^{\nu}$ if and only if det $P^{\gamma}(\nu) \neq 0$ for all $\gamma \in \hat{K}_{0}$. 
Noting that $P^{\gamma}(2 \rho)=I$ for all $\gamma \in \hat{K}_{0}$ we see that $\operatorname{det} P^{\gamma}(\nu) \neq 0$ for any $\gamma \in \hat{K}_{0}$. Thus $\operatorname{det} P^{\gamma}(\nu) \neq 0$ for almost all $\nu$.

LeMMA 7.5. Let $s \in W(A)$ the Weyl group of a. Suppose that $A_{s}(\nu): X \rightarrow X$, $A_{s}(\nu) \circ \pi_{\nu}(u)=\pi_{s(\nu-\rho)+\rho}(u) \circ A_{s}(\nu), u \in U(\mathrm{~g})$ and $A_{s}(\nu) \cdot 1_{\nu}=1_{s(\nu-\rho)+\rho}$. Then if $\gamma \in \hat{K}_{0}$ and $\lambda \in\left(V_{\gamma}^{*}\right)^{M}, v \in V_{\gamma}$,

$$
A_{s}(\nu)(\lambda \otimes v)=\lambda \circ P^{\gamma}(\nu)^{-1} P^{\gamma}(s(\nu-\rho)+\rho) \otimes v
$$

if $\operatorname{det} P^{\gamma}(\nu) \neq 0$ and $\operatorname{det} P^{\gamma}(s(\nu-\rho)+\rho) \neq 0$ for all $\gamma \in \hat{K}_{0}$.

PRoof. If $u \in H^{*}$ then $A_{s}(\nu) T_{\nu}(u)=A_{s}(\nu) \pi_{\nu}(u) 1_{\nu}=\pi_{s(\nu-\rho)+\rho}(u) A_{s}(\nu) 1_{\nu}$, $=\pi_{s(\nu-\rho)+\rho}(u) 1_{s(\nu-\rho)+\rho}=T_{s(\nu-\rho)+\rho}(u)$. Hence $A_{s}(\nu) T_{\nu} \circ T_{2 \rho}^{-1}(\lambda \otimes v)=$ $T_{s(\nu-\rho)+\rho} \circ T_{2 \rho}^{-1}(\lambda \otimes v)$. Thus

$$
\begin{aligned}
A_{s}(\nu)(\lambda \otimes v) & =\left(T_{s(\nu-\rho)+\rho} \circ T_{2 \rho}^{-1}\right) \circ\left(T_{\nu} \circ T_{2 \rho}^{-1}\right)^{-1}(\lambda \otimes v) \\
& =\lambda \circ P^{\gamma}(\nu)^{-1} P^{\gamma}(s(\nu-\rho)+\rho) \otimes v
\end{aligned}
$$

by Lemma 7.3.

If $\operatorname{dim} a=1$ we identify $\nu \in a_{C}^{*}$ with $\nu\left(H_{0}\right) \in C$ where 1 is the smallest eigenvalue of ad $\left.H_{0}\right|_{n}$.

THEOREM 7.6 (COMPARE Kostant [10]). If $G$ is split rank 1 then $\operatorname{dim} V_{\gamma}^{M}=$ 1 if $\gamma \in \hat{K}_{0}$. Hence $P^{\gamma}(\nu)$ is a scalar.

(1) If $G$ is locally isomorphic with $S O(n, 1)$ we parametrize $\hat{K}_{0}$ as in Theorem 3.2 and set $P^{\gamma}(\nu)=P_{j}(\nu)$ if $\gamma$ is the class of $\mathcal{F}^{j}$. Then $P_{j}(\nu)=c_{j} \Pi_{i=0}^{j-1}(\nu+j)$, $c_{j} \neq 0$.

(2) If $G$ is locally isomorphic with $S U(n, 1)$ then let $P^{\gamma}(\nu)=P_{i, j}(\nu)$ if $\gamma$ is the class of $\mathcal{S C}^{i \mathrm{~J}}$. Then

$$
P_{i, j}(\nu)=c_{i j} \prod_{k=0}^{i-1}(2 k+\nu) \prod_{k=0}^{j-1}(2 k+\nu) .
$$

(3) If $G$ is locally isomorphic with $S p(n, 1)$ then let $P^{\gamma}(\nu)=P_{i, j}(\nu)$ if $\gamma$ is the class of $V^{i j}$ (see Theorem 3.2). Then

$$
P_{i j}(\nu)=c_{i j} \prod_{l=0}^{(i-j) / 2-1}(\nu+2 l-2) \prod_{l=0}^{(i+j) / 2-1}(\nu+2 l) .
$$

(4) If $G$ is locally isomorphic with $F_{4}$ with $K=\operatorname{Spin}(9)$ then if $\gamma$ is the class of $V^{i j}$,

$$
P_{i, j}(\nu)=c_{i j} \prod_{l=0}^{(i-j) / 2-1}(2 l-6+\nu) \prod_{l=0}^{(i+j) / 2-1}(2 l+\nu) .
$$

(See [7, Theorem 3.1] for notation.) 
Proof. Suppose $\operatorname{dim} a=1$. If $S\left(p_{C}\right)$ is the symmetric algebra of $p_{C}$, then we look at $S\left(p_{\mathrm{C}}\right)$ as the polynomials on $\mathcal{P}^{*}$. Using $B$, the killing form of $g$, $\mathscr{P} *$ is equivalent to $\mathscr{P}$ as a representation of $K$ under Ad. Also, if $H_{0}$ is as above, then $\operatorname{Ad}(K) \cdot H_{0}=K / M$. Thus $S\left(p_{\mathrm{C}}\right) \mid K / M=X$ as a representation of $K$. Let $\mathcal{H}$ be the harmonic polynomials in $S\left(p_{\mathcal{C}}\right)$. Then if $\lambda: S(g) \rightarrow U(g)$ is the symmetrization mapping $\lambda(\mathcal{H C})=H^{*}$. Let $\mathcal{K}_{\gamma}$ be an irreducible component of $\mathcal{X}$ labeled by its equivalence class. If $f \in \mathcal{Y}_{y}^{M}$ then $\pi_{v}(\lambda(f)) 1_{v}=$ $P^{\gamma}(\nu) f$ as a function on $K / M$. We therefore see

$$
\text { If } f \in \mathcal{H}_{\gamma}^{M}, f \neq 0 \text {, then } \operatorname{deg} P^{\gamma}=\operatorname{deg} f .
$$

The result now follows from Lemma 7.5, Theorem 6.1 and [7, Theorem 5.2] by observing that the $a_{\gamma}(\nu)$ are of the form $n_{\gamma}(\nu) / d_{\gamma}(\nu)$ with $n_{\gamma}(\nu)$ and $d_{\gamma}(\nu)$ relatively prime and $\operatorname{deg} d_{\gamma}(\nu)=\operatorname{deg} f$ for $f \neq 0, f \in \mathcal{F}_{\gamma}^{M}$. Q.E.D.

8. Remarks on square-integrability. In this section we will examine the representations of $\$ 7$ to determine which of these representations are squareintegrable. We first however will make some general statements concerning the square-integrability of representations.

Let $G, K, A$ and $a$ be as in $\$ 2$. Let $a^{+}=\{H \in a: \alpha(H)>0$ for all $\alpha \in \Delta^{+}$. Suppose $\pi: G \rightarrow \mathfrak{L}(\mathcal{F}, \mathcal{F C})$ is an irreducible representation of $G$. Let $\nu$ be an irreducible representation of $K$ which occurs in $\pi$ and let $v_{1}, \ldots, v_{m}$ be orthonormal vectors which span a vector space which is irreducible under the restriction of $\pi$ to $K$ and transforms according to $\nu$.

$\pi$ is said to be square-integrable if, for all $\varphi$ and $\psi$ in $\mathcal{K}, \int_{G}\left\langle\left.\langle\pi(g) \varphi, \psi\rangle\right|^{2} d g\right.$ $<\infty$. From Harish-Chandra [3], we know that $\pi$ is square-integrable if and only if there exist $\varphi$ and $\psi$ nonzero in $\mathcal{H}$ such that

$$
\int_{G}|\langle\pi(g) \varphi, \psi\rangle|^{2} d g<\infty \text {. }
$$

Thus $\pi$ is square-integrable if and only if

Now

$$
\int_{G}\left|\left\langle\pi(g) v_{1}, v_{1}\right\rangle\right|^{2} d g<\infty .
$$

$$
\int_{G}\left\langle\pi(g) v_{1}, v_{1}\right\rangle d g=\int_{K^{+}} \int_{A^{+}} \int_{K}\left|\left\langle\pi\left(k_{1}\right) \pi(a) \pi\left(k_{2}\right) v_{1}, v_{1}\right\rangle\right|^{2} \delta(a) d k_{1} d a d k_{2}
$$

where $\delta(a)=\Pi_{\alpha \in \Delta^{+}}\left(e^{\alpha(\log a)}-e^{-\alpha(\log a)}\right)^{m_{\alpha}}$ (see Helgason [5, p. 382]).

From Schur's lemma, we have

$$
\begin{aligned}
\int_{K} \int_{A^{+}} \int_{K} \delta(a)\left|\left\langle\pi\left(k_{1}\right) \pi(a) \pi\left(k_{2}\right) v_{1}, v_{1}\right\rangle\right|^{2} d k_{2} d a d k_{2} \\
\quad=\int_{K} \int_{A^{+}} \int_{K} \delta(a) \sum_{j, k=1}^{m}\left|v_{j 1}\left(k_{1}\right)\right|^{2}\left|v_{l 1}\left(k_{2}\right)\right|^{2}\left\langle\pi(a) v_{j}, v_{l}\right\rangle d k_{1} d a d k_{2} \\
\quad=\frac{1}{m^{2}} \int_{A^{+}} \delta(a) \sum_{j, l=1}^{m}\left|\left\langle\pi(a) v_{j}, v_{l}\right\rangle\right|^{2} d a .
\end{aligned}
$$


Thus $\pi$ is square-integrable if and only if for all $1 \leqslant j, l \leqslant m$

$$
\int_{A^{+}} \delta(a)\left|\left\langle\pi(a) v_{j}, v_{l}\right\rangle\right|^{2} d a<\infty
$$

Hence, from the formula for $\delta(a)$, $\pi$ is square-integrable if and only if for all $1<j, l<m$

$$
\int_{A^{+}} e^{2 \rho(\log a)}\left|\left\langle\pi(a) v_{j}, v_{l}\right\rangle\right|^{2} d a<\infty
$$

From Harish-Chandra [4], we know that if $\mathcal{H}$ contains a $K$-fixed vector, $\pi$ is not square-integrable.

We now suppose that $G$ is a classical split rank one group. That is, $G / K$ is the open unit ball in $\mathbf{F}^{n}$. Our main results of this section can now be stated.

THEOREM 8.1. (1) (Takahashi [17]). If $\mathbf{F}=\mathbf{R}$ and $n>3$ the unitary representation $\pi_{-k}$ induces on the completion of $X^{-k} / V_{k}$ is not square-integrable.

(2a) If $\mathbf{F}=\mathbf{C}$ and $n \geqslant 2$ the unitary representations $\pi_{0}$ induces on the completion of $\mathrm{H}_{0}^{+} / \mathrm{L}_{0}$ and $\mathrm{H}_{0}^{-} / \mathrm{L}_{0}$ are not square-integrable.

(2b) If $\mathbf{F}=\mathbf{C}$ and $n \geqslant 3$ the unitary representation $\pi_{-2 l}$ induces on the completion of $\mathrm{X}^{-2 l} /\left(\mathrm{H}_{2 l}^{+}+\mathrm{H}_{2 l}^{-}\right)$is not square-integrable.

(3) If $\mathbf{F}=\mathbf{H}$ and $n \geqslant 3$ the unitary representation $\pi_{-2 l}$ induces on the completion of $X^{-2 l} / M_{l}$ is not square-integrable, and the unitary representation $\pi_{2}$ induces on the completion of $X^{2} / T$ is not square-integrable.

Proof. Since $G$ in each of these cases has split rank $1, \operatorname{dim} a=1$. So if we let

$$
a_{t}=\left(\begin{array}{c|c|c}
\cosh t & 0 & \sinh t \\
\hline 0 & I & 0 \\
\hline \sinh t & 0 & \cosh t
\end{array}\right) \in A
$$

we have that

$$
\int_{A^{+}} \delta(a) f(a) d a=c_{0} \int_{0}^{\infty} \delta\left(a_{t}\right) f\left(a_{t}\right) d t
$$

where $c_{0}>0$ and $f \in C_{c}\left(A^{+}\right)$.

Proof of (1). Observe that the $K$-irreducible subspace $\mathcal{F}^{k+1}$ occurs in $X^{-k} / V_{k}$. Since $n \geqslant 3$ there is a harmonic polynomial of degree $k+1$ which is independent of the first variable $x_{1}$. (Recall the coordinates of $\mathbf{F}^{n}$.)

Now

$$
\begin{aligned}
\pi_{-k}\left(a_{t}\right) G\left(x_{2}, \ldots, x_{n}\right) \\
=\left(\cosh t-\sinh t x_{1}\right)^{k} G\left(\frac{x_{2}}{\cosh t-\sinh t x_{1}}, \ldots, \frac{x_{n}}{\cosh t-\sinh t x_{1}}\right) \\
=\frac{1}{\cosh t-\sinh t x_{1}} G\left(x_{2}, \ldots, x_{n}\right) .
\end{aligned}
$$


If the representation $\pi_{-k}$ induces on the completion of $X^{-k} / V_{k}$ were square-integrable we would have

$$
\int_{0}^{\infty} \delta\left(a_{t}\right)\left|\left\langle\pi_{-k}\left(a_{t}\right) G, G\right\rangle\right|^{2} d t<\infty
$$

where

$$
\left\langle\pi_{-k}\left(a_{t}\right) G, G\right\rangle=\int_{S^{n-1}} \pi_{-k}\left(a_{t}\right) G\left(x_{1}, \ldots, x_{n}\right) \overline{G\left(x_{1}, \ldots, x_{n}\right)} d \mu\left(S^{n-1}\right)
$$

and $d \mu\left(S^{n-1}\right)$ is the standard measure on $S^{n-1}$. However,

$$
\begin{aligned}
& \int_{S^{n-1}} \pi_{-k}\left(a_{t}\right) G\left(x_{1}, \ldots, x_{n}\right) \overline{G\left(x_{1}, \ldots, x_{n}\right)} d \mu\left(S^{n-1}\right) \\
& \quad=\int_{S^{n-1}} \frac{1}{\cosh t-(\sinh t) x_{1}}\left|G\left(x_{2}, \ldots, x_{n}\right)\right|^{2} d \mu\left(S^{n-1}\right) \\
&=\frac{1}{\cosh t} \int_{S^{n-1}} \frac{1}{1-(\tanh t) x_{1}}\left|G\left(x_{2}, \ldots, x_{n}\right)\right|^{2} d \mu\left(S^{n-1}\right) \\
&> \frac{1}{2 \cosh t} \int_{S^{n-1}}\left|G\left(x_{2}, \ldots, x_{n}\right)\right|^{2} d \mu S^{n-1}=\frac{A}{2 \cosh t} .
\end{aligned}
$$

Since $2 \rho\left(\log a_{t}\right)=(n-1) t$ and $e^{((n-1) / 2) t}(A / 2 \cosh t) \sim A e^{((n-3) / 2) t}$, for large $t$ we see that our representation is not square-integrable if $n>3$.

Proof of (2). (a) Observe that $\mathcal{F}^{1,0}$ occurs in $H_{0}^{+} / L_{0}$ and $\mathcal{F}^{0,1}$ occurs in $H_{0}^{-} / L_{0}$.

Let $G\left(z_{1}, \ldots, z_{n}\right)=z_{2}$ and let $a_{t}$ be as above. Then

$$
\pi_{0}\left(a_{t}\right) G\left(z_{1}, \ldots, z_{n}\right)=\frac{z_{2}}{\cosh t-(\sinh t) z_{1}} .
$$

Then

$$
\begin{aligned}
\left\langle\pi_{0}\left(a_{t}\right) G, G\right\rangle & =\int_{S^{2 n-1}} \frac{\left|z_{2}\right|^{2}}{\cosh t-(\sinh t) z_{1}} d \mu\left(S^{2 n-1}\right) \\
& =\frac{1}{\cosh t} \int_{S^{2 n-1}} \frac{\left|z_{2}\right|^{2}}{1-(\tanh t) z_{1}} d \mu\left(S^{2 n-1}\right) \\
& =\frac{1}{\cosh t} \int_{S^{2 n-1}}\left|z_{2}\right|^{2} d \mu\left(S^{2 n-1}\right)=\frac{A}{\cosh t} .
\end{aligned}
$$

As $2 \rho\left(\log a_{t}\right)=2 n t$ and $e^{n t}(A / \cosh t) \sim 2 A e^{(n-1) t}$ for large $t$, we see that the unitary representation $\pi_{0}$ induces on the completion of $H_{0}^{+} / L_{0}$ is not square-integrable. The case of $H_{0}^{-} / L_{0}$ is now obvious. Thus we have proved (2a).

(2b) Observe that $\mathcal{I}^{l+1, l+1}$ occurs in $X^{-2 l} /\left(H_{2 l}^{+}+H_{2 l}^{-}\right)$. Recall that if $G$ is in $\mathfrak{K}^{l+1, l+1}$, 


$$
G\left(\alpha z_{1}, \ldots, \alpha z_{n}\right)=|\alpha|^{2 l+2} G\left(z_{1}, \ldots, z_{n}\right) \text { for } \alpha \in \mathbf{C} \text {. }
$$

Let $G \in \mathcal{H}^{l+1, l+1}$ which is independent of $z_{1}$ and $\bar{z}_{1}$. This can clearly be done since $n>3$. Then an easy calculation gives

$$
\pi_{-2 l}\left(a_{t}\right) G\left(z_{1}, \ldots, z_{n}\right)=\frac{1}{\left|\cosh t-(\sinh t) z_{1}\right|^{2}} G\left(z_{2}, \ldots, z_{n}\right) .
$$

Moreover,

$$
\begin{aligned}
\left\langle\pi_{-2 l}\left(a_{t}\right) G, G\right\rangle & \\
& =\int_{S^{2 n-1}} \frac{1}{\left|\cosh t-(\sinh t) z_{1}\right|^{2}}\left|G\left(z_{2}, \ldots, z_{n}\right)\right|^{2} d \mu\left(S^{2 n-1}\right) \\
& =\frac{1}{\cosh ^{2} t} \int_{S^{2 n-1}} \frac{1}{\left|1-(\tanh t) z_{1}\right|^{2}}\left|G\left(z_{2}, \ldots, z_{n}\right)\right|^{2} d \mu\left(S^{2 n-1}\right) \\
& =\frac{1}{\cosh ^{2} t} \int_{S^{2 n-1}} \frac{1}{1-(\tanh t)^{2}\left|z_{1}\right|^{2}}\left|G\left(z_{2}, \ldots, z_{n}\right)\right|^{2} d \mu\left(S^{2 n-1}\right) \\
& >\frac{1}{\cosh ^{2} t} \int_{S^{2 n-1}}\left|G\left(z_{2}, \ldots, z_{n}\right)\right|^{2} d \mu\left(S^{2 n-1}\right) \\
& =\frac{A}{\cosh ^{2} t} \quad \text { with } A>0 .
\end{aligned}
$$

As $e^{n t}\left(A / \cosh ^{2} t\right) \sim 4 A e^{(n-2) t}$ for large $t$ and $n>3$ the representation $\pi_{-2 t}$ induces on the completion of $\mathrm{X}^{-2 l} /\left(\mathrm{H}_{2 l}^{+}+\mathrm{H}_{2 l}^{-}\right)$is not square-integrable.

Proof of (3). Observe that the $K$-irreducible space $V^{2 l+4,0}$ occurs in $X^{-2 l} / M_{l}$ and the $K$-irreducible space $V^{2,0}$ occurs in $X^{2} / T$. We first need a characterization of the elements of $V^{2 m, 0}$ and this is provided easily as follows:

(i) Let $\overline{\mathcal{S}}^{2 m}=\left\{f \in \mathcal{P}^{2 m}: f\left(w_{1} w, \ldots, w_{n} w\right)=|w|^{2 m} f\left(w_{1}, \ldots, w_{n}\right)\right.$ for all $w \in \mathbf{F}=\mathbf{H}\}$. An easy calculation shows that $\overline{\mathscr{\rho}}^{2 m}$ is $K=(\operatorname{Sp}(n) \times S p(1))-$ invariant.

(ii) Observe from Theorem 4.1 that $\left(V^{2 m j}\right)^{M} \cap \overline{\mathcal{P}}^{2 m} \neq(0)$ if and only if $l=0$. Thus $V^{2 m, 0} \subset \bar{\rho}^{2 m}$.

Now let $G \in V^{2 l+4,0}$ which is independent of the real coordinates of $w_{1}$. This can be done since $n>3$. Then

$$
\pi_{-2 l}\left(a_{t}\right) G\left(w_{1}, \ldots, w_{n}\right)=\frac{1}{\left|\cosh t-(\sinh t) w_{1}\right|^{4}} G\left(w_{2}, \ldots, w_{n}\right) .
$$

Similarly, if $G \in V^{2,0}$ which is independent of the real coordinates of $w_{1}$

$$
\pi_{2}\left(a_{t}\right) G\left(w_{1}, \ldots, w_{n}\right)=\frac{1}{\left|\cosh t-(\sinh t) w_{1}\right|^{4}} G\left(w_{2}, \ldots, w_{n}\right) .
$$

Now 


$$
\begin{aligned}
& \int_{S^{4 n-1}} \frac{1}{\left|\cosh t-(\sinh t) w_{1}\right|^{4}}\left|G\left(w_{2}, \ldots, w_{n}\right)\right|^{2} d \mu\left(S^{4 n-1}\right) \\
& =\frac{1}{\cosh ^{4} t} \int_{S^{4 n-1}} \frac{1}{\left|1-(\tanh t) w_{1}\right|^{4}}\left|G\left(w_{2}, \ldots, w_{n}\right)\right|^{2} d \mu\left(S^{4 n-1}\right) .
\end{aligned}
$$

Now

$$
\frac{1}{\left|1-(\tanh t) w_{1}\right|^{4}}=\frac{1}{\left(1-(\tanh t)\left(w_{1}+\bar{w}_{1}\right)+\left|w_{1}\right|^{2} \tanh ^{2} t\right)^{2}}
$$

using the coordinates $\left|w_{1}\right|^{2}=\cos ^{2} \xi$ and $w_{1}+\bar{w}_{1}=2 \cos \xi \cos t(0<\xi<$ $\pi / 2,0<t<\pi)$ we have

$$
\frac{1}{\left|1-(\tanh t) w_{1}\right|^{4}}=\sum_{k=0}^{\infty}(k+1) \frac{(2 \tanh t)^{k} \cos ^{k} \xi \cos ^{k} t}{\left(1+\cos ^{2} \xi \tanh ^{2} t\right)^{k+2}} .
$$

Using the fact that $\int_{0}^{\pi} \cos ^{k} t \sin ^{2} t d t-0$ for odd $k$, we obtain from Theorem 4.1(4) that

$$
\begin{aligned}
& \frac{1}{\cosh ^{4} t} \int_{S^{4 n-1}} \frac{1}{\left|1-(\tanh t) w_{1}\right|^{4}}\left|G\left(w_{2}, \ldots, w_{n}\right)\right|^{2} d \mu\left(S^{4 n-1}\right) \\
& =\frac{1}{\cosh ^{4} t} \sum_{k=0}^{\infty} \int_{S^{4 n-1}}(2 k+1) \frac{(\tanh t)^{2 k}\left(w_{1}+\bar{w}_{1}\right)^{2 k}}{\left(1+\left|w_{1}\right|^{2} \tanh ^{2} t\right)^{2 k+2}} \\
& \quad \cdot\left|G\left(w_{2}, \ldots, w_{n}\right)\right|^{2} d \mu\left(S^{4 n-1}\right) \\
& >\frac{1}{\cosh ^{4} t} \int_{S^{4 n-1}} \frac{1}{\left(1+\left|w_{1}\right|^{2} \tanh ^{2} t\right)^{2}}\left|G\left(w_{2}, \ldots, w_{n}\right)\right|^{2} d \mu\left(S^{4 n-1}\right) \\
& >\frac{1}{4 \cosh ^{4} t} \int_{S^{4 n-1}}\left|G\left(w_{2}, \ldots, w_{n}\right)\right|^{2} d \mu\left(S^{4 n-1}\right) \\
& =\left(\frac{A}{4}\right) \frac{1}{\cosh ^{4} t} \quad \text { with } A>0 .
\end{aligned}
$$

As $2 \rho\left(\log a_{t}\right)=(4 n+2) t$ and $e^{(2 n+1) t}(A / 4)\left(1 / \cosh ^{4} t\right) \sim 4 A e^{(2 n-3) t}$ for large $t$ and $n>3$, (3) now follows. This completes the proof of the theorem.

Using the fact that no unitary representation that contains a $K$-fixed vector is square-integrable, we have:

COROLlARY 1. No unitary representation which arises from the spherical principal series (irreducible or reducible) of $S O(n, 1), S U(n, 1)$ or $S p(n, 1)$ for $n>3$ is square-integrable. 
We now consider the groups $S O(n, 1), S U(n, 1)$ and $S p(n, 1)$ for $n \leqslant 2$. In the case of $S U(1,1)$ or $S O(2,1)$ the discrete series does arise when the principal series is reducible; see [1] or [14]. Now $\operatorname{Sp}(1,1)$ is a covering of $S O^{\circ}(4,1)$ and is thus dealt with above. $S O^{\circ}(1,1)=\mathbf{R}$ and no more need be said about it.

It remains only to consider the groups $S U(2,1)$ and $S p(2,1)$. The following proposition shows that the breakdown in the proof of Theorem 8.1 for these groups is more than mere technical inconvenience.

THEOREM 8.2. Let $G=S U(2,1)$; then the unitary representation that $\pi_{-2 k}$ induces on the completion of $\mathrm{X}^{-2 k} /\left(\mathrm{H}_{-2 k}^{+}+\mathrm{H}_{-2 k}^{-}\right), \mathrm{H}_{-2 k}$, is square-integrable.

The proof of this theorem requires a little preparation. We will actually get more precise estimates than will be necessary to prove Theorem 8.2. Let $T_{1}$ be the subgroup of $K$ consisting of matrices in $K$ of the form

$$
\left[\begin{array}{lll}
e^{i \theta} & 0 & 0 \\
0 & e^{-i \theta} & 0 \\
0 & 0 & 1
\end{array}\right]
$$

Then $T_{1}$ is a maximal torus of the subgroup $K_{1}$ consisting of all matrices in $K$ of the form

$$
\left[\begin{array}{l|l}
g & 0 \\
\hline 0 & 1
\end{array}\right]
$$

with $g, 2 \times 2$. Noting that $K$ is a central extension of $K_{1}$ we see that the representation of $K_{1}$ on $\mathcal{F}^{k, k}$ is already irreducible. Furthermore standard theory says that $\mathcal{H}^{k, k}=\sum_{j=-k}^{k} \mathcal{F}_{2 j}^{k, k}$ with

(a) $\operatorname{dim} \mathcal{F}_{2 j}^{k, k}=1,-k \leqslant j \leqslant k$.

(b) If $f \in \mathcal{F}_{2 j}^{k, k}$ and if

$$
k_{1}(\theta)=\left[\begin{array}{lll}
e^{i \theta} & 0 & 0 \\
0 & e^{-i \theta} & 0 \\
0 & 0 & 1
\end{array}\right]
$$

then $\pi_{0}\left(k_{1}(\theta)\right) f=e^{-i 2 j \theta} f$.

Let $P: \mathscr{P} \rightarrow \mathcal{H}$ be the harmonic projection (see the discussion preceding Theorem 4.1).

Set $\phi_{k, j}\left(z_{1}, z_{2}\right)=P\left(\left|z_{2}\right|^{2 k-2 j} z_{1}^{j} \bar{z}_{2}^{j}\right)$ for $0 \leqslant j \leqslant k$. Set $\phi_{k,-j}=\overline{\phi_{k, j}}$.

LEMMA 8.1. $\mathcal{H}_{j}^{k, k}=\mathbf{C} \phi_{k, j}$.

Proof. Clearly $\phi_{k, j} \in \mathcal{F}_{j}^{k, k}$. It is thus only necessary to show that $\phi_{k, j} \neq 0$. We leave this as a (simple) exercise to the reader. 
LEMMA 8.2. $\Delta\left(\left|z_{2}\right|^{2 k-2 j} z_{1}^{j} \bar{z}_{2}^{j}\right)=4 k(k-j)\left|z_{2}\right|^{2 k-2 j-2} z_{1}^{j} \bar{z}_{2}^{j}$.

The proof is again a straightforward computation. Using Lemma 8.2 one concludes directly.

LEMMA 8.3. $\phi_{k, j}=z_{1}^{j} \bar{z}_{2}^{j} \sum_{i=0}^{k-j} c_{k j, i}\left|z_{2}\right|^{2 k-2 j-2 i} r^{2 i}$ for $0 \leqslant j \leqslant k$; here $c_{k j, k} \in \mathbf{R}$, $c_{k, j, 0}=1$.

LEMMA 8.4. (1) $\left\langle\pi_{-2 k}\left(a_{t}\right) \phi_{k+1, j}, \phi_{k+1, l}\right\rangle=0$ if $j \neq l$.

(2) $\left\langle\pi_{-2 k}\left(a_{t}\right) \phi_{k+1, j}, \phi_{k+1, j}\right\rangle=\operatorname{conj}\left(\left\langle\pi_{-2 k}\left(a_{t}\right) \phi_{k+1,-j}, \phi_{k+1,-j}\right\rangle\right)$ for $-k \leqslant j$ $<k($ here $\operatorname{conj}(z)=\bar{z})$.

(3) If $0<j \leqslant k+1$ then

$$
\left\langle\pi_{-2 k}\left(a_{t}\right) \phi_{k+1, j}, \phi_{k+1, j}\right\rangle=(\cosh t)^{-(2+j)} \int_{S^{3}} \frac{z_{1}^{j} \bar{z}_{2}^{j}\left|z_{2}\right|^{2 k-2 j+2} \overline{\phi_{k+1, j}(z)}}{1-(\tanh t)^{2}\left|z_{1}\right|^{2}} d \mu .
$$

Proof. To prove (1) we note that the centralizer in $G$ of the set of all $a_{t}$, $t \in \mathbf{R}$ is $M$, the group of all matrices of the form

$$
m(\theta)=\left[\begin{array}{lll}
e^{i \theta} & 0 & 0 \\
0 & e^{-2 i \theta} & 0 \\
0 & 0 & e^{i \theta}
\end{array}\right]
$$

Now $\pi_{0}(m(\theta)) \phi_{k, j}=e^{-3 j \theta} \phi_{k, j}$. Thus (1) follows from Schur orthogonality. (2) follows from the fact that $\pi_{-2 k}\left(a_{t}\right)$ is a real operator $\operatorname{conj}\left(\left(\pi_{-2 k}\left(a_{t}\right) f\right)(z)\right)$ $=\left(\pi_{-2 k}\left(a_{t}\right) \bar{f}\right)(z)$ for $z \in S^{3}$.

(3) Let $0<j<k+1$.

$$
\begin{aligned}
\left(\pi_{-2 k}\left(a_{t}\right) \phi_{k+1, j}\right)(z) & \\
= & {\left[\frac{1-(\tanh t)^{2}}{\left|1-(\tanh t) z_{1}\right|^{2}}\right]^{-(2 k / 2)} \phi_{k+1, j}\left(\left(\cosh t-(\sinh t) z_{1}\right)^{-1}\right.} \\
& \left.\cdot\left((\cosh t) z_{1}-\sinh t, z_{2}\right)\right) \\
= & \left|1-(\tanh t) z_{1}\right|^{2 k}(\cosh t)^{2 k}(\cosh t)^{-2 j}\left|1-(\tanh t) z_{1}\right|^{-2 j} \\
& \cdot\left((\cosh t) z_{1}-\sinh t\right)^{j} z_{2}^{j} \\
& \cdot\left\{\begin{array}{l}
k-j+1 \\
\sum_{i=0} c_{k, j}(\cosh t)^{-2 k-2+2 j+2 i}
\end{array}\right. \\
= & \left|1-(\tanh t) z_{1}\right|^{-2}(\cosh t)^{-2}\left((\cosh t) z_{1}-\sinh t\right)^{j} \bar{z}_{2}^{j}\left|z_{2}\right|^{2 k+2-2 j} \\
& +r^{2} \psi_{t}\left(z_{1}, z_{2}\right) \quad \text { with } \psi_{t}\left(z_{1}, z_{2}\right) \in \rho^{2 k} .
\end{aligned}
$$


Hence

$$
\begin{aligned}
\left\langle\pi_{-2 k}\left(a_{t}\right) \phi_{k+1, j}, \phi_{k+1, j}\right\rangle & (\cosh t)^{-2} \int_{S^{3}} \frac{\left((\cosh t) z_{1}-\sinh t\right)^{j} \bar{z}_{2}^{j}\left|z_{2}\right|^{2 k+2-2 j}}{\left|1-(\tanh t) z_{1}\right|^{2}} \overline{\phi_{k+1, j}(z)} d \mu \\
= & (\cosh t)^{-2} \sum_{p, q>0}(\tanh t)^{p+q} \sum_{l=0}^{j}\left(\begin{array}{l}
j \\
l
\end{array}\right)(-1)^{l}(\cosh t)^{j-l}(\sinh t)^{l} \\
& \cdot \int_{S^{3}} z_{1}^{p+j-l} \bar{z}_{1}^{q} \bar{z}_{2}^{j}\left|z_{2}\right|^{2 k+2-2 j} \frac{\phi_{k+1, j}(z)}{\phi^{2}} d \mu(z) .
\end{aligned}
$$

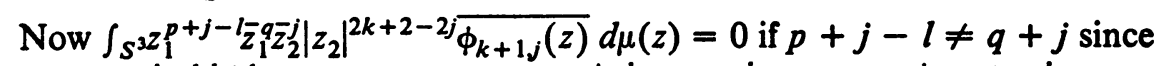
$\phi_{k+1, j} \in \mathcal{F}^{k+1, k+1}$. Thus noting $(\cosh t)^{j-l}(\sinh t)^{l}=(\cosh t)^{j}(\tanh t)^{l}$,

$$
\begin{aligned}
\left\langle\pi_{-2 k}\left(a_{l}\right) \phi_{k+1, j}, \phi_{k+1, j}\right\rangle & \\
= & (\cosh t)^{-2}(\cosh t)^{j} \sum_{l=0}^{j}\left(\begin{array}{l}
j \\
l
\end{array}\right)(-1)^{l} \sum_{q=0}^{\infty}(\tanh t)^{2 q+2 l} \\
& \cdot \int_{S^{3}}\left|z_{1}\right|^{2 q} z_{1}^{j} \bar{z}_{2}^{j}\left|z_{2}\right|^{2 k+2-2 j} \overline{\phi_{k+1, j}(z)} d \mu(z)=(\cosh t)^{-2}(\cosh t)^{j} \\
& \cdot \sum_{l=0}^{j}\left(\begin{array}{l}
j \\
l
\end{array}\right)(-1)^{l}(\tanh t)^{2 l} \int_{S^{3}} \frac{z_{1}^{j} \bar{z}_{2}^{j}\left|z_{2}\right|^{2 k+2-2 j} \overline{\phi_{k+1, j}(z)}}{1-(\tanh t)^{2}\left|z_{1}\right|^{2}} d \mu .
\end{aligned}
$$

The lemma now follows from the formula $1-(\tanh t)^{2}=(\cosh t)^{-2}$.

LEMMA 8.5. $\int_{S^{3}}\left|z_{1}\right|^{2 p}\left|z_{2}\right|^{2 q} d \mu(z)=p ! q ! /(p+q+1)$ !.

Proof. This result is standard (cf. Vilenkin [18]). One can give a very elementary proof of the lemma by noting

$$
\Delta^{p}\left|z_{1}\right|^{2 p}=4^{p}(p !)^{2}, \quad \Delta^{p} r^{2 p}=4^{p}(p+1) ! p ! .
$$

Hence $\int_{S^{3}}\left|z_{1}\right|^{2 p} d \mu(z)=1 /(p+1)$. The result can then be proven by induction using

$$
\left|z_{1}\right|^{2 p}\left|z_{2}\right|^{2 q+2}=\left|z_{1}\right|^{2 p}\left|z_{2}\right|^{2 q} r^{2}-\left|z_{1}\right|^{2 p+2}\left|z_{2}\right|^{2 q}
$$

LEMMA 8.6. (1) $\frac{1}{2}<\int_{S^{3}}\left(\left|z_{2}\right|^{2} /\left(1-(\tanh t)^{2}\left|z_{1}\right|^{2}\right)\right) d \mu(z)<1$ for all $t$ and there is a positive constant $c_{2}$ such that

(2) $1<\int_{S^{3}}\left(1 /\left(1-(\tanh t)^{2}\left|z_{1}\right|^{2}\right)\right) d \mu(z) \leqslant c_{2}|t|$ for $|t|$ sufficiently large.

Proof. The lower estimates are trivial and (1) follows from the inequality $1-(\tanh t)^{2}\left|z_{1}\right|^{2}>\left|z_{2}\right|^{2}$.

We now prove (2). As 


$$
\begin{aligned}
\int_{S^{3}} \frac{1}{1-(\tanh t)^{2}\left|z_{1}\right|^{2}} & d \mu(z)=\sum_{p=0}^{\infty}(\tanh t)^{2 p} \int_{S^{3}}\left|z_{1}\right|^{2 p} d \mu(z) \\
& =\sum_{p=0}^{\infty}(\tanh t)^{2 p} \frac{p !}{p+1}<1+\sum_{p=1}^{\infty} \frac{(\tanh t)^{2 p}}{p} \\
& =1-(\tanh t)^{-2} \log \left(1-(\tanh t)^{2}\right)<c_{2}|t|
\end{aligned}
$$

for $|t|$ sufficiently large.

Lemma 8.7. Let $c_{2}$ be as in Lemma 8.6. Then

(1)

$$
\begin{aligned}
& \frac{(\cosh t)^{-(3+k)}((k+1) !)^{2}}{(2 k+3) !} \\
& \quad<\left\langle\pi_{-2 k}\left(a_{t}\right) \phi_{k+1, \pm(k+1)}, \phi_{k+1, \pm(k+1)}\right\rangle<(\cosh t)^{-(3+k)} .
\end{aligned}
$$

(2) If $0<|j|<k$ then

$$
\left|\left\langle\pi_{-2 k}\left(a_{t}\right) \phi_{k+1, j}, \phi_{k+1, j}\right\rangle\right|<c_{2}|t|(\cosh t)^{-(2 k+4-|j|)}\left\|\phi_{k+1, j}\right\|_{\infty}
$$

for $|t|$ sufficiently large. Here

$$
\left\|\phi_{k+1, j}\right\|_{\infty}=\sup _{z \in S^{3}}\left|\phi_{k+1, j}(z)\right| \text {. }
$$

Proof. (1)

$$
\begin{aligned}
\left\langle\pi_{-2 k}\left(a_{t}\right) \phi_{k+1, k+1}, \phi_{k+1, k+1}\right\rangle & \\
& =(\cosh t)^{-(3+k)} \int_{S^{3}} \frac{z_{1}^{k+1} \bar{z}_{2}^{k+1} \frac{\phi_{k+1, j}(z)}{1-(\tanh t)^{2}\left|z_{1}\right|^{2}}}{1-(z)} \\
& =(\cosh t)^{-(3+k)} \int_{S^{3}} \frac{\left|z_{1}\right|^{2 k+2}\left|z_{2}\right|^{2 k+2}}{1-(\tanh t)^{2}\left|z_{1}\right|^{2}} d \mu(z) \\
& >(\cosh t)^{-(3+k)} \int_{S^{3}}\left|z_{1}\right|^{2 k+2}\left|z_{2}\right|^{2 k+2} d \mu(z) \\
& =(\cosh t)^{-(3+k)} \frac{((k+1) !)^{2}}{(2 k+3) !} .
\end{aligned}
$$

On the other hand,

$$
\int_{S^{3}} \frac{\left|z_{1}\right|^{2 k+2}\left|z_{2}\right|^{2 k+2}}{1-(\tanh t)^{2}\left|z_{1}\right|^{2}} d \mu(z)<\int_{S^{3}} \frac{\left|z_{2}\right|^{2}}{1-(\tanh t)^{2}\left|z_{1}\right|^{2}} d \mu(z)<c_{2} .
$$

This proves (1).

To prove (2) we first observe that if $s>1$, then 
(a)

$$
\int_{S^{3}} \frac{z_{1}^{j} z_{2}^{j}\left|z_{2}\right|^{2 s}\left|z_{1}\right|^{2 l} \overline{\phi_{s+l+j, j}(z)}}{1-(\tanh t)^{2}\left|z_{1}\right|^{2}} d \mu(z)
$$

$$
=-(\cosh t)^{-2} \int_{S^{3}} \frac{z_{1}^{j} z_{2}^{j}\left|z_{2}\right|^{2 s-2}\left|z_{1}\right|^{2 l+2} \overline{\phi_{s+l+j j}(z)}}{1-(\tanh t)^{2}\left|z_{1}\right|^{2}} d \mu(z) .
$$

Indeed,

$$
\begin{aligned}
& \int_{S^{3}} \frac{z_{1}^{j} \bar{z}_{2}^{j}\left|z_{2}\right|^{2 s}\left|z_{1}\right|^{2 l} \overline{\phi_{s+l+j j}(z)}}{1-\left|z_{1}\right|^{2}} d \mu(z) \\
& \quad=\int_{S^{3}} z_{1}^{j} \bar{z}_{2}^{j}\left|z_{2}\right|^{2 s-2}\left|z_{1}\right|^{2 l} \overline{\phi_{s+l+j j}(z)} d \mu(z)=0 .
\end{aligned}
$$

Since $\operatorname{deg}\left(z_{1}^{j} \bar{z}_{2}^{j}\left|z_{2}\right|^{2 s-2}\left|z_{1}\right|^{2 l}\right)=2 s+2 j+2 l-2, \operatorname{deg}\left(\phi_{s+l+j j}\right)=2 s+2 l+2 j$ and $\phi_{s+l+j, j}$ is harmonic. This implies that

$$
\begin{aligned}
& \int_{S^{3}} \frac{z_{1}^{j} \bar{z}_{2}^{j}\left|z_{2}\right|^{2 s}\left|z_{1}\right|^{2 l} \overline{\phi_{s+l+j j}(z)}}{1-(\tanh t)^{2}\left|z_{1}\right|^{2}} d \mu(z) \\
& \quad=\int_{S^{3}} z_{1}^{j} \bar{z}_{2}^{j}\left|z_{2}\right|^{2 s}\left|z_{1}\right|^{2 l} \overline{\phi_{s+l+j j}(z)}\left\{\frac{1}{1-(\tanh t)^{2}\left|z_{1}\right|^{2}}-\frac{1}{1-\left|z_{1}\right|^{2}}\right\} d \mu(z) .
\end{aligned}
$$

This yields (a) after the appropriate algebraic manipulation.

Now suppose that $0<j<k$.

$$
\begin{aligned}
\left\langle\pi_{-2 k}\left(a_{t}\right) \phi_{k+1, j},\right. & \left.\phi_{k+1, j}\right\rangle \\
= & (\cosh t)^{-(2+j)} \cdot \int_{S^{3}} \frac{z_{1}^{j} \bar{z}_{2}^{j}\left|z_{2}\right|^{2 k-2 j+2} \overline{\phi_{k+1, j}(z)}}{1-(\tanh t)^{2}\left|z_{1}\right|^{2}} d \mu(z) \\
= & (\cosh t)^{-(2+j)}(\cosh t)^{-(2 k-2 j+2)}(-1)^{k-j+1} \\
& \cdot \int_{S^{3}} \frac{z_{1}^{j} \bar{z}_{2}^{j}\left|z_{1}\right|^{2 k-2 j+2} \overline{\phi_{k+1, j}(z)}}{1-(\tanh t)^{2}\left|z_{1}\right|^{2}} d \mu(z)
\end{aligned}
$$

by repeated application of (a). Thus

$$
\begin{aligned}
\left|\left\langle\pi_{-2 k}\left(a_{t}\right) \phi_{k+1, j}, \dot{\phi_{k+1, j}}\right\rangle\right| & \\
& =(\cosh t)^{-(4+2 k-j)} \int_{S^{3}} \frac{z_{1}^{j} \bar{z}_{2}^{j}\left|z_{2}\right|^{2 k-2 j+2} \overline{\phi_{k+1, j}(z)}}{1-(\tanh t)^{2}\left|z_{1}\right|^{2}} d \mu(z) \\
& <(\cosh t)^{-(4+2 k-j)}\left\|\phi_{k+1, j}\right\|_{\infty} \int_{S^{3}} \frac{\left|z_{1}\right|^{\prime}\left|z_{2}\right|^{j}\left|z_{1}\right|^{2 k-2 j+2}}{1-(\tanh t)^{2}\left|z_{1}\right|^{2}} d \mu(z) \\
& <(\cosh t)^{-(4+2 k-j)}\left\|\phi_{k+1, j}\right\|_{\infty} \int_{S^{3}} \frac{1}{1-(\tanh t)^{2}\left|z_{1}\right|^{2}} d \mu(z) \\
& <c_{2}|t|(\cosh t)^{-(4+2 k-j)}\left\|\phi_{k+1, j}\right\|_{\infty} .
\end{aligned}
$$


The result for $-k+1<j<0$ follows from the observation

$$
\left|\left\langle\pi_{-2 k}\left(a_{t}\right) \phi_{k+1,-j}, \phi_{k+1,-j}\right\rangle\right|=\left|\left\langle\pi_{-2 k}\left(a_{t}\right) \phi_{k+1, j}, \phi_{k+1, j}\right\rangle\right| \text {. Q.E.D. }
$$

PROOF OF THEOREM 8.2. If $G=S U(2,1)$ then $e^{2 p\left(\log a_{1}\right)}=e^{4 t}$. On the other hand, Lemma 8.7 implies

$$
\left|\left\langle\pi_{-2 k}\left(a_{t}\right) \phi_{k+1, j}, \phi_{k+1, j}\right\rangle\right|<e^{-3 t}
$$

for $t$ sufficiently large, $k \geqslant 0$, and $0 \leqslant|j|<k+1$. Thus

$$
\int_{0}^{\infty} e^{4 t}\left|\left\langle\pi_{-2 k}\left(a_{t}\right) \phi_{k+1, j}, \phi_{k+1, j}\right\rangle\right|^{2} d t<\infty
$$

for all $k \geqslant 0$ and $0 \leqslant|j| \leqslant k+1$. The result now follows from the observation preceding Theorem 8.1.

In order to handle $\operatorname{Sp}(2,1)$ we will need the weight space characterization of spherical harmonics given in $\$ 2$. We consider the representation $\pi_{-2 l}$ induces on $X^{-2 l} / M_{l}$ and the representation $\pi_{2}$ induces on $X^{2} / T$. Let $T_{0}$ be a compact Cartan subgroup of $S p(2,1)$ in $S p(2) \times S p(1)$. Let $\Theta_{-2 l}$ and $\Theta_{2}$ denote the restrictions of the respective characters of these representations to $T_{0}$.

If $P_{0}$ denotes the set of positive compact roots and

$$
D_{0}=\prod_{\alpha \in P_{0}}\left(e^{\alpha / 2}-e^{-\alpha / 2}\right)
$$

we have that formally

$$
\Theta_{-2 l}=\frac{1}{D_{0}} \sum_{p>l+2} \sum_{q>0} \sum_{s \in W}(\operatorname{det} s) \exp s\left[(p+1) \lambda_{1}+(q+1)\left(\lambda_{2}+\lambda_{3}\right)\right]
$$

where $W$ is the Weyl group of $S p(2) \times S p(1)$. Thus, if we let $P_{n}$ be the positive noncompact roots and set $D_{+}=\Pi_{\alpha \in P_{n}}\left(e^{\alpha / 2}-e^{-\alpha / 2}\right)$ we have that

$$
\Theta_{-2 l}=\frac{1}{D_{0} D_{+}} \sum_{s \in W}(\operatorname{det} s) \exp s\left[(l+2) \lambda_{1}+\lambda_{2}+\lambda_{3}\right]
$$

and also

$$
\Theta_{2}=\frac{1}{D_{0} D_{+}} \sum_{s \in W}(\operatorname{det} s) \exp s\left[\lambda_{1}+\lambda_{2}+\lambda_{3}\right]
$$

Again from Harish-Chandra [4] and Schmid [20] we have that $\Theta_{-2 l}$ and $\Theta_{2}$ agree on $T_{0}$ with discrete series representations. We must add that since we have not verified that the characters of our unitary representations for $S p(2,1)$ are tempered we cannot conclude that our representations are discrete series representations.

We close with a brief remark on integrable representations which we believe is well known but we give a proof for completeness sake. 
Proposition 8.2. Let $G$ be a semisimple Lie group with finite center and let $G=K \bar{A}^{+} K$ as above. Let $\pi: G \rightarrow \mathcal{L}(\mathcal{H}, \mathcal{H})$ be an irreducible unitary representation of $G$. Then, if $V$ is an irreducible representation of $K$ which occurs in $\mathcal{H}$, we have that $\pi$ is integrable if and only if for all $\varphi$ and $\psi$ in $V$

$$
\int_{A^{+}} \delta(a)|\langle\pi(a) \varphi, \psi\rangle| d a<\infty .
$$

Proof. Clearly, if $\int_{A^{+}} \delta(a)|\langle\pi(a) \varphi, \psi\rangle| d a<\infty$ for all $\varphi$ and $\psi$ in $V, \pi$ is integrable by Harish-Chandra [3].

Suppose that $\pi$ is integrable. Then

$$
\int_{G}|\langle\pi(x) \varphi, \psi\rangle| d x=\int_{K} \int_{K^{+}} \int_{A^{+}} \delta(a)\left|\left\langle\pi(a) \pi\left(k_{1}\right) \varphi, \pi\left(k_{2}\right) \psi\right\rangle\right| d a d k_{1} d k_{2}<\infty .
$$

Let $F\left(k_{1}, k_{2}\right)(a)=\left\langle\pi(a) \pi\left(k_{1}\right) \varphi, \pi\left(k_{2}\right) \psi\right\rangle$. Then

$$
\int_{A^{+}} \delta(a)\left|F\left(k_{1}, k_{2}\right)(a)\right| d a<\infty
$$

for almost all $\left(k_{1}, k_{2}\right)$ in $K \times K$. Then, if $S$ is the subset of $K \times K$ for which (*) holds, the linear span of $\left\{F\left(k_{1}, k_{2}\right) \mid\left(k_{1}, k_{2}\right) \in S\right\}$ is the same as the linear span of $\left\{F\left(k_{1}, k_{2}\right) \mid\left(k_{1}, k_{2}\right) \in K \times K\right\}$. Thus our result holds.

COROLlaRY 1. The representations $\left(\pi_{0}, H_{0}\right),\left(\pi_{1}, H_{1}\right)$ are not integrable. The representations $\left(\pi_{-2 k}, H_{-2 k}\right)$ for $k>1$ are integrable.

Proof. Lemma 8.7(1) implies that if $h=0$ or 1 ,

$$
\int_{0}^{\infty} e^{4 t}\left|\left\langle\pi_{-2 k}\left(a_{t}\right) \phi_{k+1, k+1}, \phi_{k+1, k+1}\right\rangle\right| d t=\infty
$$

If $k>2$ then Lemma 8.7 implies that

$$
\left|\left\langle\pi_{-2 k}\left(a_{\imath}\right) \phi_{k+1, j}, \phi_{k+1, j}\right\rangle\right| \leqslant C e^{-5 t}
$$

for $t$ large and $0<|j|<k+1$. We are left to analyze $k=2$. If $k=2$ then $\left|\left\langle\pi_{-4}\left(a_{t}\right) \phi_{3, \pm 3}, \phi_{3, \pm 3}\right\rangle\right|<C e^{-5 t}$ and

$$
\left|\left\langle\pi_{-4}\left(a_{t}\right) \phi_{3, j}, \phi_{3, j}\right\rangle\right|<C_{2}|t| e^{-(8-|j|) t}\left\|\phi_{3, j}\right\|_{\infty} \text { for }|t| \text { large. Q.E.D. }
$$

Note. If $G=S U(2,1)$ and $T$ is a maximal torus of $K$, then it is easily seen that the representations of $\left(\pi_{-2 k}, H_{-2 k}\right)$ for $k>0$ are not $T$-finite. Hence they do not belong to the holomorphic discrete series. (See Enright, Thesis, University of Washington at Seattle.)

\section{BIBLIOGRAPHY}

1. I. M. Gel'fand, M. I. Graev and N. Ja. Vilenkin, Generalized functions. Vol. 5: Integral geometry and representation theory, Fizmatgiz, Moscow, 1962; English transl., Academic Press, New York, 1966. MR 28 \#3324; 34 \#7726.

2. Harish-Chandra, Representations of semisimple Lie groups. II, Trans. Amer. Math. Soc. 76 (1954), 26-65. MR 15, 398. 
3. , Representations of semisimple Lie Groups. VI: Integrable and square-integrable representations, Amer. J. Math. 78 (1956), 564-628. MR 18, 490.

4. _ Discrete series for semisimple Lie groups. II. Explicit determination of the characters, Acta Math. 116 (1966), 1-111. MR 36 \#2745.

5. S. Helgason, Differential geometry and symmetric spaces, Academic Press, New York, 1962. MR 26 \#2986.

6.,$A$ duality for symmetric spaces with applications to group representations, Advances in Math. 5 (1970), 1-154. MR 41 \#8587.

7. K. Johnson, Composition series and intertwining operators for spherical principal series. II, Trans. Amer. Math. Soc. 215 (1976), 269-283.

8. K. Johnson and N. Wallach, Composition series and intertwining operators for the spherical principal series, Bull. Amer. Math. Soc. 78 (1972), 1053-1059. MR 46 \#9238.

9. A. W. Knapp and E. M. Stein, Intertwining operators for semisimple groups, Ann. of Math. 93 (1971), 489-578.

10. B. Kostant, On the existence and irreducibility of certain series of representations, Bull. Amer. Math. Soc. 75 (1969), 627-642. MR 39 \# 7031.

11. B. Kostant and S. Rallis, Orbits and representations associated with symmetric spaces, Amer. J. Math. 93 (1971), 753-809. MR 47 \#399.

12. R. A. Kunze and E. M. Stein, Uniformly bounded representations. III. Intertwining operators for the principal series on semisimple groups, Amer. J. Math. 89 (1967), 385-442. MR 38 \#269.

13. E. Nelson, Analytic vectors, Ann. of Math. (2) 70 (1959), 572-615. MR 21 \#5901.

14. P. J. Sally, Jr., Intertwining operators and the representations of $S L(2, \mathbf{R})$, J. Functional Analysis 6 (1970), 441-453. MR 45 \#8775.

15. G. Schiffmann, Intégrales d'entrelacement et fonctions de Whittaker, Bull. Soc. Math. France 99 (1971), 3-72. MR 47 \#400.

16. A. I. Stern, Completely irreducible representations of SU(2, 1), Dokl. Akad. Nauk SSSR 179 (1968), 1289-1292 = Soviet Math. Dokl. 9 (1968), 560-564. MR 37 \#4203.

17. R. Takahashi, Sur les représentations unitaires des groupes de Lorentz généralisés, Bull. Soc. Math. France 91 (1963), 289-433. MR 31 \#3544.

18. N. Ja. Vilenkin, Special functions and the theory of group representations, Izdat. "Nauka", Moscow, 1965; English transl., Transl. Math. Monographs, Vol. 22, Amer. Math. Soc., Providence, R. I., 1968. MR 35 \#420; 37 \#5429.

19. E. T. Whittaker and G. N. Watson, $A$ course of modern analysis, Cambridge Univ. Press, New York, 1927; reprint, 1962. MR 31 \#2375.

20. W. Schmid, On a conjecture of Langlands, Ann. of Math. (2) 93 (1971), 1-42. MR 44 \#4149.

21. Harish-Chandra, Spherical functions on a semisimple Lie group. I, Amer. J. Math. 80 (1958), 241-310. MR 20 \# 925.

22. N. R. Wallach, Application of the higher osculating spaces to the spherical principal series, J. Differential Geometry 5 (1971), 405-413. MR 45 \#8778.

Department of Mathematics, Rutgers University, University Heights Campus, New BRUNSWICK, NEW JERSEY 08903 (Current address of N. R. Wallach)

Current address (K. D. Johnson): Department of Mathematics, Indiana University, Bloomington, Indiana 47401 